\title{
TGIF2-Induced HMGB3 Promotes Esophageal Squamous Cell Carcinoma Progression Through TGF $\beta$ Signaling
}

Liaoran Niu

Xijing Hospital

Wanli Yang

Xijing Hospital

Wei Zhou

Xijing Hospital

Lili Duan

Xijing Hospital

Xiaoqian Wang

Xijing Hospital

Yiding Li

Xijing Hospital

Qi Wang

Xijing Hospital

Chengchao Xu

94719 military hospital

Yujie Zhang

Xijing Hospital

Jinqiang Liu

Xijing Hospital

Qingchuan Zhao

Xijing Hospital

Liu Hong ( $\nabla$ hongliufmmu@163.com )

Fourth Military Medical University

Daiming Fan

Xijing Hospital

\section{Research}

Keywords: Esophageal Squamous Cell Carcinoma, HMGB3, TGIF2, TLR3, TGF- $\beta$ 
Posted Date: August 31st, 2021

DOl: https://doi.org/10.21203/rs.3.rs-800914/v1

License: (c) (i) This work is licensed under a Creative Commons Attribution 4.0 International License. Read Full License 


\section{Abstract}

Background: Proliferation and metastasis are the major malignant phenotypes of esophageal squamous cell carcinoma (ESCC) and the main causes for poor survival in patients with ESCC. Nevertheless, the underlying mechanisms of ESCC proliferation and metastasis remains unclear. The high mobility group box protein family 3 (HMGB3) is one of the HMGB family members. It is critically involved in the occurrence and development of various carcinomas. However, the knowledge of HMGB3 in ESCC remains limited. In this study, we elucidated the role of HMGB3 in ESCC proliferation and metastasis, and the concrete mechanism.

Methods: Expression level of HMGB3 and TGF- $\beta$ interacting factor 2 (TGIF2) in ESCC cell lines and tissues was quantified by qRT-PCR, Western Blot, and immunohistochemistry. In vitro and in vivo assays revealed the functions of TGIF2 and HMGB3 in ESCC. RNA-seq was performed to search for the downstream signaling of HMGB3. ChIP assay and were performed to explore the relationship of HMGB3 and TGIF2. HMGB3-interacting protein was validated by immunoprecipitation.

Results: Higher expression of TGIF2 and HMGB3 was observed in ESCC cell lines and tissues and was associated with worse prognosis of ESCC patients. TGIF2 and HMGB3 upregulation could promote ESCC proliferation and metastasis, and vice versa. TGIF2 and HMGB3 upregulation can activate Smaddependent TGF- $\beta$ signaling. TGIF2 can transcriptionally regulate HMGB3, and its TGF- $\beta$ inducing capability and oncogenic role are at least partly HMGB3-dependent. Additionally, TLR3 was identified as a client protein of HMGB3, and their combination might be the reason of TGF- $\beta$ activation.

Conclusions: Collectively, HMGB3-dependent TGIF2 overexpression activates TGF- $\beta$ signaling and promotes the proliferation and metastasis of ESCC via TLR3 regulation. These findings revealed that TGIF2 and HMGB3 could be prognostic indicators of ESCC and targeting TGIF2/HMGB3/TLR3 axis might improve the OS of ESCC patients.

\section{Background}

Esophageal squamous cell carcinoma (ESCC) is the seventh most prevalent cancer and has the sixthhighest cancer-associated mortality rate wordwide [1,2]. Although improvement in earlier diagnosis and effective treatment have enhanced the overall survival (OS) rate of ESCC patients, long-term survival of ESCC patients remains poor because of unlimited proliferation and distant metastasis [3]. ESCC patients' five-year survival rates are less than $20 \%$ [4]. Multiple studies were devoted to disclose the underlying mechanisms of ESCC but their results were far from satisfactory [4]. Therefore, continuous and in-depth investigation of the mechanisms of ESCC and establishing novel therapeutic strategies are still in urgent need.

HMGB3 belongs to the high mobility group box protein family (HMGB1, 2, 3), and they share similar amino acids sequence and identical functional regions [5]. Abundant studies have reported the concrete mechanisms of HMGB1 and 2 in ESCC [6, 7], while HMGB3 was less investigated [7]. Recent studies 
showed that high expression of HMGB3 was closely related to poor prognosis of gastric cancer (GC) [8], HCC[9], and ESCC [10]. They also highlighted the role of HMGB3 in proliferation [11], metastasis [12], hypoxia [13] and drug resistance [14, 15]. HMGB3 can exert its function through the mTOR [16], Wnt [17], and DNA damage signaling pathways [14]. It can also activate other molecules, including but not limited to hypoxia-inducible factor 1a (HIF1a) [18], HOXA transcript at the distal tip (HOTTIP) [18], and matrix metallopeptidase 7 (MMP7) [17]. Further in-depth investigation of HMGB3 may be helpful to understand the HMGB family and may provide new insight into the improvement of ESCC prognosis.

TGF- $\beta$ interacting factor 2 (TGIF2) belongs to the TGIF family [19]. TGIF2 can act as transcriptional repressors by interacting with SMADs in TGF- $\beta$ pathways $[20,21]$. It can also bind to the promotor of targeted DNAs and act as either a transcriptional activator or a repressor independent of TGF- $\beta$ [20]. TGIF2 was previously confirmed to regulate CDH1 [22], FUT8 [23], and OCT4 [24] as a transcription factor. Thus, it can assist the malignant transformation of CRC [22], melanoma [23], and lung adenocarcinoma (LUAD) [24]. The expression of TGIF2 and HMGB3 are positively associated in pro-B cells, and they both donate to the self-renewal of fetal-B cells [25]. However, the characteristic of TGIF2 and the relationship between HMGB3 and TGIF2 in carcinoma have not been investigated.

TGF- $\beta$ can combine with its receptor and activate the downstream Smad-dependent or Smad-independent signaling, resulting in ligand-induced transcription [26] [27]. Almost all human cell types are responsive to TGF- $\beta$ signaling [28]. TGF- $\beta$ signaling can regulate cell proliferation, differentiation, survival, adhesion, and cellular microenvironment to maintain homeostasis and inhibit the incipient tumors from malignancy [28]. TGF- $\beta$ was confirmed to be upregulated in ESCC patients compared with the asymptomatic patients [29]. Moreover, TGF- $\beta$ signaling can promote the tumorigenesis of ESCC by inducing Epithelialmesenchymal transition (EMT) [30], angiogenesis [31], chemoresistance [32], and immunological disorder [33]. However, to clearly verify its characteristic in ESCC still needs further investigation. TGIF2 and HMGB1 were all reported to be associated with the regulation of TGF- $\beta$ signaling[22, 34-36]. TGIF2 is universally accepted as a co-repressor of R-Smads (the compound of Smad2, Smad3, and Smad4) [20], and its transcriptional function can be attenuated when EMT occurs after activation of TGF- $\beta$ signaling [22]. HMGB1 can interact with its receptor TLR4 and subsequently activate TGF- $\beta$ pathway [34]. However, the relationship between HMGB3, TGIF2 and TGF- $\beta$ has not been investigated.

This study highlighted the oncogenic role of TGIF2 and HMGB3 in the proliferation and metastasis of ESCC. They are both highly expressed in ESCC and their overexpression is closely related to the poor prognosis of ESCC patients. In addition, both TGIF2 and HMGB3 lead to the activation of TGF- $\beta$ signaling. Mechanistically, TGIF2 transcriptionally regulate HMGB3 and its malignancy inducing capability, and TGF- $\beta$ activating capability is partly HMGB3-dependent. Collectively, this study firstly confirmed the role of HMGB3, TGIF2 in ESCC progression and the concrete mechanism involved in this. This study improves the understanding of function of HMGB3, TGIF2 as well as the relationship between them. Targeting TGIF2/HMGB3/TGF- $\beta$ axis may be promising for the treatment of ESCC.

\section{Materials And Methods}




\subsection{Cell culture}

Human ESCC cell lines EC9706 and EC109 and ECA109 were maintained in our lab (Xian, China). het-1A, a human immortal esophageal squamous epithelial cell line, was donated by Zhengzhou University (Zhengzhou, China). ESCC cells and het-1A were cultured in Roswell Park Memorial Institute (RPMI)-1640 medium (Gibco, Thermo Fisher Scientific, Cambridge, MA, USA) supplemented with $10 \%$ fetal bovine serum (Evergreen; Hangzhou, China), $100 \mu \mathrm{g} / \mathrm{ml}$ of streptomycin, and $100 \mathrm{U} / \mathrm{ml}$ of penicillin in the medium (HyClone; Logan, Utah, USA). All cells were cultured in a humid incubator at $37^{\circ} \mathrm{C}$ with $5 \% \mathrm{CO}_{2}$.

\subsection{Total RNA extraction and qRT-PCR}

We implemented total RNA isolation and extraction from the tissue samples and cell lines using Trizol reagent (Invitrogen, Waltham, MA, United States). cDNA was obtained by reverse transcription of the RNA using the PrimeScript RT Reagent Kit (TaKaRa, Tokyo, Japan). qRT-PCR was subsequently performed to examine the expression levels of HMGB3 and TGIF2 by the SYBR Premix Ex Taq II Kit (TaKaRa, Tokyo, Japan). GAPDH was used as an internal standard. The $2^{-\triangle \triangle C t}$ method was used to calculate the relative mRNA expression. The primer sequences were listed below. HMGB3 forward: 5'-

CCATGATTCCTTCATATTTGC-3'; reverse: 5'-GTAATACGGTTATCCACGCG-3'; TGIF2 forward:

5'- TACTTGCACCGCTACAACGC -3'; reverse: 5'- TCCTTCCGAAGCATGTCTGG -3'; GAPDH forward: 5'GACAGTCAGCCGCATCTTCT-3'; reverse: 5'-GCGCCCAATACGACCAAATC-3'.

\subsection{Western blot (WB) assay}

The cells were lysed using radioimmunoprecipitation assay (RIPA) buffer (Beyotime Biotechnology, Shanghai, China) added with protease repressors (Millipore, Temecula, CA, USA) on ice. Then, the cell lysates were centrifuged at 12,000 g for 15 min. A BCA Protein Assay Kit (Pierce, Rockford, IL, United States) was used to detect the concentration of total proteins. Equal volumes of proteins were separated, employing $10 \%$ sodium dodecyl sulfate-poly acrylamide gel electrophoresis (SDS-PAGE). Then, they were transferred onto nitrocellulose membranes (Millipore, Temecula, CA, USA). Subsequently, membranes were blocked with $5 \%$ non-fat milk for one hour at $37^{\circ} \mathrm{C}$ and then incubated with antibodies overnight at $4^{\circ} \mathrm{C}$. After that, the membranes were incubated with horseradish peroxidase (HRP)-labeled secondary antimouse IgG or anti-rabbit IgG antibodies (Abcam,Cambridge, MA, USA) for one hour at $37^{\circ} \mathrm{C}$. The following primary antibodies were applied to exam the proteins expression: anti-HMGB3 (\#ab75782, Abcam, Cambridge, MA, USA), anti-TGIF2 (\#ab190152, Abcam,Cambridge, MA, USA), anti-SMAD2 (\#5339, Cell Signaling Technology, Cambridge, MA, USA), anti-p-SMAD2 (\#3108, Cell Signaling Technology, Beverly, MA, USA), anti-SMAD3 (\#9523, Cell Signaling Technology, Beverly, MA, USA), anti-p-SMAD3 (\#9520, Cell Signaling Technology, Beverly, MA, USA), anti-SMAD2/3 (\#8685, Cell Signaling Technology, Beverly, MA, USA), anti-TLR3 (\#ab62566, Abcam, Cambridge, MA, USA), anti-TGF- $\beta$ (\#ab215715, Abcam, Cambridge, MA, USA) and anti- $\beta$-actin (\#3700, Cell Signaling Technology, Beverly, MA, USA). Protein bands visualization was implemented on an enhanced chemiluminescence detection system (BioRad, CA, USA) and analyzed by image $J$ software. 


\subsection{Plasmid construction and chromatin immunoprecipitation assay}

5 -flanking deletion and site-directed mutation were constructed. The sequence from -2000bp to 500 around the promoter site of human HMGB3 was cloned into the pGL3-Basic vector (Promega). The HMGB3 promoter construct (-2000/500) was used as a template to generate 5'-flanking deletion constructs of the HMGB3 promoter. We validated the promoter fragments by DNA sequencing.

Magna ChIP G assay kit (Millipore, Temecula, CA, USA) was employed for ChIP assay performance. Briefly speaking, utilizing $1 \%$ formaldehyde, indicated cell lines were crosslinked at $37^{\circ} \mathrm{C}$ for $10 \mathrm{~min}$, followed by glycine quenching. The bound DNA was subsequently obtained from the sonicated cell lysates. The bound DNA was thereafter coimmunoprecipitated with primary antibodies against TGIF2 (Santa cruz, sc-390870, CA, USA) and normal IgG (Cell Signaling Technology, Danvers, MA, USA). Then the corresponding binding sites of the promoters was amplified after subjected to PCR.

\subsection{Cell transfection and infection}

Negative control lentiviral vectors and lentiviral vectors for upregulating or downregulating the target genes were designed and provided by Genechem Co. Ltd. (Shanghai, China). Sequences for the shRNAs were as follows: shHMGB3-1 (93473-1), CCGGCAGATAAAGTGCGCTATGACTCGAGTCATAGCGCACTTTATCTGTTTTTG; shHMGB3-2 (93474-1), CCGGGAGGCAAGAAGAAGAAGGACTCGAGTCCTTCTTCTTCTTGCCTCTTTTTT; shTGIF2-1(94886-1), CCGGCCATCCCTTTAGTCTCTGAAACTCGAGTTTCAGAGACTAAAGGGATGGTTTTT. shTGIF2-2(94887-1), CCGGGACCCTAATCAGTTTACCATTCTCGAGAATGGTAAACTGATTAGGGTCTTTTT.

ESCC cells were infected with the MOl set as 40 . The cells were then selected using $1-4 \mathrm{ug} / \mathrm{ml}$ puromycin for 14 days.

\subsection{CCK-8 assay}

$1 \times 10^{3}$ cells in $100 \mu$ l were seeded into 96 -well plates per well. We replaced the original medium with CCK8 solution (TransDetect Cell Counting Kit, Transgene, Beijing, China) with complete medium mixing at a $1: 9$ ratio at the same time in 5 days. We subsequently incubated cells at $37^{\circ} \mathrm{C}$ for three hours. Each sample was inspected at $450 \mathrm{~nm}$, and a microplate reader (Bio-Rad, CA, USA) was used to obtain the absorbance.

\subsection{In vitro migration and invasion assays}

In vitro migration and invasion abilities of infected cell lines were measured using 24-well Transwells ( $8 \mu \mathrm{m}$ pore size, Corning, Inc., NY, USA). In the migration experiment, $4 \times 10^{5}$ cells were seeded in the top chamber. While in the invasion experiment, every top chamber was coated with $200 \mathrm{mg} / \mathrm{ml} \mathrm{Matrigel}$ and dried for one hour at $37^{\circ} \mathrm{C}$. Then, $8 \times 10^{5}$ cells were plated in the chamber, and the invading and migrating cells at the lower layer were counted $36 \mathrm{~h}$ later. 


\subsection{Colony formation assay}

The ESCC cells suspension (800 cells) was seeded in 6-well plates. They were then cultured for two weeks in a $5 \% \mathrm{CO}_{2}$ incubator at $37 \circ \mathrm{C}$. Subsequently, the cells were fixed with $10 \%$ formalin for $15 \mathrm{~min}$, after which they were stained via $0.1 \%$ crystal violet for $15 \mathrm{~min}$.

\subsection{Cell cycle assay}

The seeded stable infected ESCC cells attaining the log phase via trypsinization were harvested to $1 \mathrm{ml}$ centrifuge tube. Subsequently, we rinsed cells in a phosphate-buffered saline (PBS) buffer. We then fixed the samples in $75 \%$ ethanol for 1 hour at $-20^{\circ} \mathrm{C}$. Fixed cells were rinsed again by PBS, followed by $400 \mathrm{ul}$ propidium iodide (Servicebio,) staining within 100 ul RNase at $37^{\circ} \mathrm{C}$ for 30 minutes in the dark. We finally implemented cell cycle analysis by the flow cytometer (CytoFLEX, Beckman Coulter, Brea, CA, United States).

\subsection{Xenograft experiment and in vivo metastasis experiment}

Balb/c mice ( 6 weeks old) were obtained from Shanghai Experimental Animal Center of the Chinese Academy of Sciences (Shanghai, China). The mice were raised in specific pathogen-free conditions in the Animal Research and Care Committee of the Fourth Force Military Medical University. We randomly assigned these mice into experimental or control groups. We created the xenograft tumor models via subcutaneous injection of $5 \times 10^{6}$ indicated cells. We measured the tumor size every three days, and calculated the volume using the following formula: volume $=($ length $\times$ width2)/2. Mice were sacrificed when the volume of the largest tumor in the group is close to 1000 . We conducted tail vein injection assays in BALB/C nude mice ( 5 weeks old) via $4 \times 106$ indicated cells. Mice were sacrificed after 6 weeks when they were in poor condition, and the lungs and livers were extracted for histological staining and examination. The survival time of mice was also recorded. The use of live animals in this research was approved by the Committee inTeaching and Research (CULATR) at Fourth Military Medical University.

\subsection{Immunohistochemistry}

The human ESCC tissue microarray (TMA) (Lot No. HEsoS180Su08) was purchased from Shanghai Outdo Biotech Co., LTD (Shanghai, China). It contained 114 ESCC and 66 para-tumor tissues. These tissues were obtained from patients who underwent radical esophagectomy from April 2006 to December 2008. This research was ratified by the ethics committee of Xijing Hospital, Fourth Military Medical University. Xylene and then graded alcohol were utilized to deparaffinize the TMA slides. The endogenous peroxidase activity was blocked using $3 \% \mathrm{H}_{2} \mathrm{O}_{2}$, and $10 \%$ goat plasma was used to block the slices. Thereafter, overnight anti- HMGB3 antibody (1:800, Abcam, Cambridge, MA, United States), anti- TGIF2 antibody (1:500, Abcam, Cambridge, MA, United States) incubation was performed. Following conjugation with biotinylated secondary antibody and then HRP, DAB staining and subsequently hematoxylin counterstaining was applied. The images were obtained on a fluorescence microscope (3DHISTECH, Sysmex, Switzerland). 
Pathologists were invited to view the TMA utilizing the histochemical score (H-score) to assess the staining intensity and the percentage of stained cells. The staining intensity was scored on a range of 0 to 3: 0 (no staining), 1 (weakly staining), 2 (moderate staining), and 3 (strong staining). The percentage of stained cells was scored as follows: 0 (no staining, 0\%), 1 (staining range, 1-25\%), 2 (staining range, 26$50 \%$ ), 3 (staining range, $51-75 \%$ ), or 4 (staining range, $76-100 \%$ ). The final $\mathrm{H}$-score was calculated by multiplying values of staining intensity and percentage of stained cells. $\mathrm{H}$-score was used to classify low or high expression.

\subsection{Co-IP assay}

EC9706 and ECA109 were lysed in the non-denaturing lysis buffer in Abcam Immunoprecipitation Kit

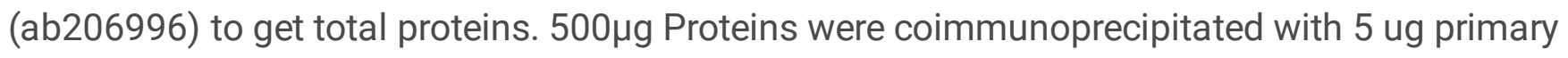
antibodies (TLR3, Novus Biologicals, NBP-24875; HMGB3, Abcam, ab75782) or IgG (Beyotime Biotechnology, Shanghai, China), and protein A/G Sepharose in the Abcam Immunoprecipitation Kit overnight at $4{ }^{\circ} \mathrm{C}$. Subsequently, the sepharose was washed for three times by wash buffer. The samples were finally boiled adding 1/4 loading buffer (Beyotime Biotechnology, Shanghai, China) before subjected to WB.

\subsection{Statistical analysis}

IBM SPSS Statistics 22.0 software was used to implement all the statistical computations. The Student's t-test was used to analyze the correlation between HMGB3 or TGIF2 expression in ESCC and para-tumor tissues. The link between the HMGB3 or TGIF2 expression and the clinicopathological parameters of ESCC patients was calculated using the Chi-square test. The Kaplan-Meier approach was then utilized to draw the survival curve of patients with different expressions of HMGB3, TGIF2, and their combinations. We performed a log-rank assessment to inspect the connection between HMGB3, TGIF2 expression and the prognosis of ESCC. two-tailed Student t-test was employed to inspect differences between variables in the variable groups. $p<0.05$ was set as significantly different.

\section{Results}

\subsection{HMGB3 is upregulated in ESCC and predict poor prognosis}

To investigate the role of HMGB3 in ESCC. We initially analyzed the gene expression level of HMGB3 in The Cancer Genome Atlas (TCGA) dataset (https://cancergenome.nih.gov/abouttcga/overview). The result showed that the expression of HMGB3 was significantly upregulated in ESCC tissues than that in adjacent tissues (Fig. 1A). Specifically, both HMGB3 mRNA and protein levels in the ESCC cell lines were critically higher than those in normal het-1A cell (Fig 1C, D). Besides, the expression of HMGB3 was analyzed in 21 pairs of ESCC tissues and adjacent nontumor tissues using qRT-PCR. As shown in Fig. 1E, mRNA expression of HMGB3 was higher in ESCC tissues than the adjacent normal control $(p=0.007)$. Additionally, the ESCC patients in the TCGA database were divided into high and low HMGB3 expression groups. The higher expression of HMGB3 was correlated with a shorter OS rate (Fig. 1B). 
Tissue microarray was also employed to further evaluate the correlation between HMGB3 expression and clinical features. The HMGB3 protein level was critically higher in ESCC tissues than in adjacent nontumorous tissues $(p<0.001)(F i g .1 F, G)$. Higher expression of HMGB3 protein is associated with a higher possibility of tumor invasion grade $(p=0.05)$ and higher American Joint Committee on Cancer (AJCC) stage $(p=0.007)$. Moreover, males tended to have higher HMGB3 expression $(p=0.046)$ (Table 1$)$. The Kaplan-Meier analysis showed that patients with HMGB3 overexpression have shorter OS rates than patients with lower HMGB3 expression (Fig. 1H).

\subsection{Overexpression of HMGB3 promotes ESCC cell proliferation, migration, and invasion in vitro}

Based on the expression level of HMGB3 in ESCC cells, we infected EC9706 with lentivirus to upregulate HMGB3 expression. We also downregulated HMGB3 expression by si-RNA in EC109 and ECA109. The expression level was confirmed by qRT-PCR (Fig. 2A) and WB (Fig. 2B). Cell Counting Kit 8 (CCK-8) assay (Fig. 2C) and colony formation assay (Fig. 2D) indicated that HMGB3 upregulation could promote the ESCC cells' proliferation, while HMGB3 downregulation attenuated the proliferative ability. Further, we conducted a cell cycle assay to disclose whether the change of cell proliferation was associated with alteration in the cell cycle profile. Consequently, the results showed that ectopic expression of HMGB3 in EC9706 attenuated G1/S arrest, while HMGB3 downregulation in EC109 and ECA109 lowered the rate of cells in the S phase (Figure 2E). Transwell assay confirmed that the upregulation of HMGB3 could promote the invasion and metastasis ability of EC9706, while HMGB3 knockdown repressed this ability (Fig. 2F).

\subsection{Overexpression of HMGB3 promotes ESCC cell proliferation, migration, and invasion in vivo.}

We established another stable cell lines by lentivirus, which suppresses HMGB3 expression in EC109 and ECA109. The result of qRT-PCR and WB showed that infection of LV- shHMGB3-1 could reduce the expression of HMGB3 mRNA and protein level in indicated cells (Fig. 3A, B). Subcutaneous xenograft nude mice models were established by stable cell lines of EC9706 with HMGB3 overexpression, ECA109 with HMGB3 knockdown and their negative control (NC) (Fig. 3C). The tumor volume and weight assessment showed that HMGB3 overexpression stimulated tumor growth, while HMGB3 downregulation suppressed tumor proliferation in vivo (Fig. 3D, E). Immunohistochemistry (IHC) staining for Ki67 and HMGB3 showed significant increases in the growth of tumor cells with high HMGB3 expression (Fig. 3F). We further performed in vivo lung metastasis experiments. The data revealed that HMGB3 upregulation shortened the OS of mice in the HMGB3 overexpression group (Fig. 3G) and enhanced ESCC cells lung metastasis incidence, while HMGB3 knockdown resulted in the opposite effect (Fig. 3H). Hematoxylin and eosin (H\&E) staining showed an increased number of metastatic lung nodules on HMGB3 overexpression, whereas HMGB3 knockdown reduced the metastatic capability (Fig. 3I). In conclusion, HMGB3 plays an oncogenic role in ESCC cell proliferation and metastasis in vivo.

\subsection{TGIF2 transcriptionally regulates HMGB3}


To investigate the concrete mechanism of HMGB3 in the tumorigenesis of ESCC, we predicted the transcriptional factors of HMGB3 in the JASPAR database (http://jaspar.genereg.net/) (Fig. 4A). The data revealed that TGIF2 might regulate HMGB3 as a transcriptional factor. A recent study also highlighted that their expression level was positively associated, and they both donate to initiate self-renewing fetal pro-B cells [25]. The correlation analysis of the TCGA database also indicated that the expression level of TGIF2 and HMGB3 might be positively associated ( $R=0.18, p=0.018)$ (Fig. 4B). To verify this hypothesis, we initially analyzed the expression of TGIF2 in ESCC tumor cell lines and normal cell het-1A by qRT-PCR (Fig. 4C) and WB (Fig. 1D). The results showed that TGIF2 expression was lower in EC9706, whereas it was higher in EC109 and ECA109. We then established stable cell lines to upregulate TGIF2 in EC9706 and suppress TGIF2 in EC109 and ECA109 by lentivirus. The expression level of TGIF2 and HMGB3 was confirmed using qRT-PCR (Fig. 4D) and WB (Fig. 4E).

Subsequently, we determined to confirm the direct relation between TGIF2 and HMGB3. Through sequence analysis, we identified two possible TGIF2-binding sites in the HMGB3 promoter (Fig. 4F). The chromatin immunoprecipitation (ChIP) showed that overexpression of TGIF2 enhanced binding of TGIF2 to binding site 1 of HMGB3 promoter, while the binding of TGIF2 to binding site 2 of HMGB3 promoter was not changed (Fig. 4F, G). We also developed and transferred plasmid to mutate the site between -2000bp to $500 \mathrm{bp}$ of HMGB3 promoter. In this progression, binding site 1 is mutated and binding site 2 remains unchanged (Fig. 4I). Luciferase activity further verified that TGIF2 overexpression could enhance the luciferase activity when binding to HMGB3 promoter. However, the mutation of the HMGB3 promoter binding site 1 inhibited this effect, indicating the binding of TGIF2 to the binding site 1 of HMGB3 promoter (Fig. 4I).

\subsection{Overexpression of TGIF2 promotes ESCC cell proliferation, migration, and invasion in vitro and in vivo.}

Using the established stable cell lines with TGIF2 overexpression or knock down as indicated, we performed CCK-8 (Fig. S1A) and colony formation assay to uncover the role of TGIF2 in ESCC cell proliferation in vitro (Fig. S1B). The results showed that TGIF2 upregulation could promote the proliferation of ESCC cells, while TGIF2 suppression delayed these malignant phenotypes. Subsequently, cell cycle assay was conducted to verify whether its expression can regulate the cell cycle progression (Fig. S1C). The assay highlighted that the role of TGIF2 in promoting proliferation may result from its ability to decrease G1/S arrest of cancer cells. The subcutaneous xenograft nude mice model was also conducted to show the role of TGIF in inducing proliferation in vivo (Fig. 5A). In the xenograft nude mice, the tumor volumes and weight of the TGIF2 overexpression group were substantially larger than those in the NC group. Reversely, tumors formed by the TGIF2 knockdown group had critically smaller volumes and lower weight than those of the NC group (Fig. 5B, C). IHC for Ki67 and TGIF2 revealed higher TGIF2 expression significantly increases the proliferation of tumor cells (Fig. 5D).

Transwell assay (Fig. S1D) showed that TGIF2 overexpression could promote the invasive and metastatic ability of ESCC cells, and vice versa. The in vivo metastasis experiment was also performed to disclose the roles of TGIF2 in influencing ESCC cells' metastatic capability in vivo. TGIF2 upregulation leads to a 
shorter OS rate of mice (Fig. 5E) and contributes to a higher rate of metastatic lung modules (Fig. 5F, G), and vice versa. Collectively, TGIF2 functions as an oncogene that promotes ESCC cell proliferation and metastasis in vitro and in vivo.

\subsection{HMGB3 is essential for TGIF2-mediated ESCC cell proliferation and metastasis.}

We further verified the role of HMGB3 in TGIF2-mediated ESCC proliferation and metastasis. We deleted HMGB3 in TGIF2-overexpressing EC9706 cells and upregulated HMGB3 in TGIF2-knockdown ECA109 cells by infection of lentivirus. The expression level of HMGB3 and TGIF2 was verified using WB (Fig. 6A).

In vitro tumorigenesis experiments including CCK-8 and colony formation assay indicated that ESCC cells' growth (Fig. S2A, B) critically decreased upon silencing of HMGB3 in EC9706 cells overexpressing TGIF2. In contrast, HMGB3 overexpression reversed the TGIF2 knock down-induced ESCC cell proliferation. Specifically, cell cycle assay showed that the critical role of HMGB3 in TGIF2-mediated proliferation resulted from its capability in inducing the increasement of S phase rate of ESCC cells (Fig. S2C). In vivo experiments (Fig. 6B) showed significant downregulation in tumor growth and tumor weight upon silencing of HMGB3 in EC9706-TGIF2 cells (Fig. 6C, D). Additionally, IHC effect for Ki67 staining was also attenuated upon silencing of HMGB3 in EC9706-TGIF2 cells (Fig. 6E).

Additionally, the Transwell assay verified the promoting role of HMGB3 in TGIF2 induced ESCC metastasis (Fig. S2D). In vivo metastatic experiment showed that HMGB3 suppression increased the OS (Fig. 6F), and reduced lung metastasis rates and the numbers of metastatic lung nodules (Fig. 6G, H) of mice in the EC9706-TGIF2 group. Thus, HMGB3 is essential for TGIF2-mediated ESCC cell proliferation and metastasis.

\subsection{Clinical significance of TGIF2 and correlation between TGIF2 and HMGB3}

To investigate the role of TGIF2 and the correlation between TGIF2 and HMGB3 in ESCC tissues, we further analyzed the mRNA expression in the 21 pairs of ESCC and normal control tissues. As revealed in Fig. 7A, TGIF2 was higher expressed in ESCC than in adjacent nontumor $(\mathrm{p}=0.0457)$. Additionally, IHC was also performed to analyze the protein expression level on the TMA (Fig. 7B). Consistently, ESCC tissues showed higher TGIF2 protein expression levels than the normal $(p=0.0087)$ (Fig. 7C). Correlation analysis confirmed the positive correlation between TGIF2 and HMGB3 $(r=0.4924, p=0.023)$ in 21 pairs samples and the TMA $(r=0.4331, p<0.001)$ (Fig. 7D, E). The Chi-square test indicated that HMGB3 overexpression positively correlated with its master regulator TGIF2 (Fig. 7F). Specifically, elevated expression of TGIF2 was closely related to higher pathological grade (Table 1). According to the K-M plotter, TGIF2 overexpression was associated with poor prognosis of ESCC patients (Fig. 7G). Besides, cooverexpression of TGIF2 and HMGB3 showed the lowest OS rate, and both of their low expression indicated the highest OS rate in the cohort of ESCC patients, suggesting that TGIF2/HMGB3 axis is critical in the prognosis of ESCC patients (Fig. $7 \mathrm{H})$. 
We further applied univariate and multivariate cox regression to investigate the prognostic value of TGIF2 and HMGB3 expression in ESCC (Table 2). The univariate analysis indicated that age $(p=0.003)$, tumor invasion ( $p=0.001)$, lymph node metastasis $(p=0.006)$, American Joint Committee on Cancer (AJCC) stage $(p<0.001)$, HMGB3 expression $(p<0.001)$ and TGIF2 expression $(p<0.001)$ were prognostic factors. The data of multivariate analysis suggested that the gender $(p=0.006)$, tumor invasion $(p=0.047)$, and HMGB3 $(p<0.001)$ expression were independent prognostic risk factors $(p<$ $.05)$.

\subsection{TGIF2 and HMGB3 can positively regulate TGF- $\beta$ signaling in ESCC}

To further identify the possible downstream target of HMGB3, we performed RNA-seq. The volcano plot and heatmap showed 168 upregulated genes and 141 downregulated ones in ECA109 cells compared with the ECA109 infected with LV-shHMGB1-1 (Fig. 8A, B). The specific expression of each gene is presented in Table S1. The Kyoto Encyclopedia of Genes and Genomes (KEGG) enrichment analysis indicated that TGF- $\beta$ signaling might be regulated by HMGB3 (Fig. 8C). To confirm the relation between HMGB3 and TGF- $\beta$ signaling, we performed WB. The data indicated that HMGB3 overexpression could enhance the expression of TGF- $\beta$, SMAD2, SMAD3, p-SMAD2, p-SMAD3, and SMAD2/3, and low expression of HMGB3 can downregulate these molecules (Fig. 8D). TGIF2 can also positively regulate TGF- $\beta$ signaling pathway in a Smad-dependent way (Fig. 8E), and this is partly HMGB3-dependent (Fig. $8 F)$. HMGB3 knockdown attenuated the inducing effect of TGIF2 overexpression on TGF- $\beta$ signaling in EC9706, while HMGB3 upregulation reversed the inhibiting function of TGIF2 knockdown in ECA109.

TLR3 was significantly overexpressed in ECA109 compared with ECA109 infected with LV-shHMGB3-1. Recent studies highlighted the relationship between HMGB and TLR family. Besides, TLR3 was reported to be involved in TGF- $\beta$ activation in various carcinomas. According to RNA-seq and recent articles, we subsequently investigated the possible relationship between HMGB3, TLR3, and TGF- $\beta$. we initially activated TLR3 using poly (I:C). Consistent with previous studies, activation of TLR3 can induce the expression of TGF- $\beta$ signaling in ESCC cell line (Fig. 8G). Moreover, we detected TLR3 in the indicated cell lines, and found that overexpression of TGIF2 and HMGB3 could enhance the expression of TLR3, and vice versa (Fig. 8D, E). Besides, HMGB3 knockdown in the EC9706-TGIF2 cells attenuated the upregulation of TLR3 and TGF- $\beta$ signaling. While upregulation of HMGB3 in the ECA109 with TGIF2 knockdown cells enhanced the TLR3 and TGF- $\beta$ signaling, indicating the role of HMGB3 in positively regulating TLR3 (Fig. 8F).

Previous study fully confirmed HMGB family and TLR family could be combined as donors and receptors to induce inflammation and carcinogenesis [12]. To further investigate the interaction between HMGB3 and TLR3, we employed a reciprocal coimmunoprecipitation (Co-IP) assay. Co-IP of the endogenous protein verified that HMGB3 could directly combine with TLR3 compare to IgG in EC9706 and ECA109. Collectively, HMGB3 can directly combine with TLR3 and subsequently activate downstream Smaddependent TGF- $\beta$ signaling (Fig. 8G). 


\section{Discussion}

Current diagnostic and therapeutic methods for ESCC have improved, and ESCC patients' OS has been prolonged [3]. However, ESCC patients' prognosis still cannot meet expectations, and unlimited proliferation and metastasis are still the main reasons for ESCC patients' poor prognosis [4]. Thus, investigation of the mechanisms underlying ESCC proliferation and metastasis is urgently needed to develop therapies. Herein, we identified that TGIF2 transcriptionally regulated HMGB3, and their expression was positively associated with ESCC proliferation and metastasis. TGIF2 and HMGB3 expression levels were relatively higher in ESCC patients, and ESCC patients with higher HMGB3 or TGIF2 levels tended to have a worse prognosis. We also found that TGIF2/HMGB3 axis can positively regulate TGF- $\beta$ signaling, and this may be related to the combination of HMGB3 and TLR3.

Similar to its family members (HMGB1 and HMGB2), HMGB3 critically participate in building nucleoprotein complexes by altering the chromatin structures, leading to the combination of multiple factors [5] and modification of these DNAs [37]. HMGB3 was associated with poor prognosis of GC [8], HCC [9] and ESCC [10]. Researches also highlighted the role of HMGB3 in proliferation [11], metastasis [12], hypoxia [13] and drug resistance [14]. However, the concrete mechanism of HMGB3 in inducing ESCC tumorigenesis was still less explored. Herein, we confirmed that HMGB3 could induce proliferation and metastasis of ESCC in vitro and in vivo. IHC also indicated that high expression of HMGB3 is associated with poor prognosis of ESCC patients, and overexpression of HMGB3 may lead to higher tumor invasion grade and higher AJCC stage. We further made an in-depth inquiry into the potential mechanism.

In previous research, HMGB3 was reported to be regulated by miRNAs including miR-200b [12], miR-2055p [11], miR-532-5p [38], and proteins including EGb 761 [39] and MMP7 [17]. We predicted possible transcriptional factors of HMGB3 employing the JASPAR database. After researching recent studies, we selected TGIF2 as an ideal target because previous studies showed that they were both overexpressed to promote the self-renewing capability in the pro-B cells [25]. Besides, sequence analysis also predicted 2 possible binding sites of HMGB3 promoter for TGIF2 (binding site 1: -1138bp -894bp and binding site 2: $-4566 \mathrm{bp} \sim-4638 \mathrm{bp}$ ). TGIF2, like TGIF1, functions by binding with R-SMAD and recruiting histone deacetylases (HADCs) or directly binding to the promoter of DNAs independent of SMADs and HADC3 [24]. TGIF2 was reported as a transcriptional activator of $\mathrm{CDH1}$, and thus it could lead to EMT of ESCC cells [22]. While Fructosyltransferase 8 (FUT8) can be transcriptionally regulated by TGIF2, leading to distant metastasis of melanoma [23]. ERK/MAPK signaling participates in the phosphorylation of TGIF2, and phosphorylated TGIF2 can transcriptionally enhance the expression of OCT4 and promote stemness of lung adenocarcinoma (LUAD) [24]. In our study, we confirmed that TGIF2 could bind to the promoter of HMGB3 and positively regulate its expression via qRT-PCR, WB, ChIP, and luciferase report. TGIF2 was reported to be involved in the progression of various carcinomas, however, the role of TGIF2 in ESCC was not investigated in previous study [23-25]. This study also firstly verified that TGIF2 can act as one oncogene in ESCC carcinogenesis. Its expression level is higher in ESCC tissues, and its high expression leads to poor prognosis of ESCC patients. Higher TGIF2 expression level may be related to higher 
pathological grade. Additionally, its overexpression can lead to proliferation and metastasis of ESCC cells, analyzed by in vitro and in vivo analysis. Specifically, the oncogenic role of TGIF2 is at least partly HMGB3-dependent.

Downstream target of HMGB3 in ESCC was also not disclosed in previous study [11, 17]. We further employed RNA-seq to underline the downstream target of HMGB3. KEGG enrichment analysis highlighted the possible relationship between HMGB3 and TGF- $\beta$ signaling. TGF- $\beta$ signaling can function through Smad-dependent or Smad-independent ways, and it plays a dual role in tumorigenesis [40]. As is generally accepted, it inhibits the malignant transformation of normal cells while inducing tumorigenesis during tumor progression [19]. The reasons for its duality are multifaceted, and the mechanisms of its tumor-inducing ability are partly disclosed, including but not limited to evasion of immune surveillance [41], myofibroblast mobilization [42], and osteoclast mobilization [43]. TGF- $\beta$ was reported to be involved in ESCC carcinogenesis $[43,45]$. Recent studies verified that TGIF2 and HMGB1 were closely related to TGF- $\beta$ signaling $[19,40]$. TGIF2 was universally accepted as a classical binding protein of R-Smad [19]. HMGB1 was also reported to positively regulate TGF- $\beta$ signaling by interacting with TLR4 and advanced glycosylation end-product specific receptor (RAGE) receptors[40]. HMGB3 can participate in mTOR [16], Wnt [17], and DNA damage signaling pathways [14]. However, whether HMGB3 could participate in TGF- $\beta$ signaling was not reported [11]. TGIF2's role in TGF- $\beta$ signaling of ESCC was also not disclosed [19]. Herein, we discovered that TGIF2 and HMGB3 could positively regulate Smad-dependent TGF- $\beta$ signaling in ESCC, and TGIF2's function in inducing TGF- $\beta$ signaling is partly HMGB3 dependent, employing WB analysis.

Given that HMGB3 could positively regulate TGF- $\beta$ signaling, we wanted to find a HMGB3 downstream target, which is also involved in the regulation of TGF- $\beta$ signaling. We observed the different expressed genes in ECA109-shHMGB3-1 and NC groups and searching through multiple studies. We then selected TLR3 for the following reasons. First, in many cases, the TLR family is essential for the inflammation and carcinoma-inducing function of HMGB1 [34, 44], which shares an identical biochemical structure with HMGB3 [2]. Consequently, TLR3, as a significant member of the TLR family, may be vital for HMGB3's participation in carcinogenesis. Second, TLR3 was certified to positively regulate TGF- $\beta$ signaling in many carcinomas, including neuroblastoma [45], breast cancer [46], and lung cancer [35]. TGF- $\beta$ is upregulated in ESCC patients and can induce tumorigenesis of ESCC [32, 33]. TLR3 may also positively regulate TGF$\beta$ in ESCC, and thus TLR3 may be a connection point between HMGB3 and TGF- $\beta$. Investigation of the relationship between HMGB3 and TLR3 can further disclose the mechanism of HMGB3 and the HMGB family. Based on the direct combination between HMGB1 and TLR4 [34, 35, 44, 47], we speculated that HMGB3 might regulate TLR3 by directly combining with it. WB indicated that TLR3 activation using poly (I:C) could induce the expression of Smad-dependent TGF- $\beta$ signaling. Additionally, TGIF2 and HMGB3 could positively regulate TLR3, and the inducing role of TGIF2 is partly HMGB3-dependent. Specifically, the Co-IP test confirmed the direct combination of HMGB3 and TLR3.

In conclusion, we verified TGIF2's role in positively regulating HMGB3 as a transcriptional factor. TGIF2 and HMGB3 were both upregulated in ESCC patients, and their overexpression indicated a poor 
prognosis. TGIF2 and HMGB3 could promote proliferation and metastasis of ESCC and induce the expression of TLR3 and TGF- $\beta$ signaling. The inducing capability of TGIF2 is at least partly HMGB3dependent. Additionally, TLR3 can activate TGF- $\beta$ signaling in ESCC, and this can be positively regulated by HMGB3. The direct combination between HMGB3 and TLR3 may be the concrete mechanism for their TGF- $\beta$ signaling-inducing capability.

Herein, we firstly investigated the oncogenic role of TGIF2 and HMGB3 in ESCC proliferation and metastasis. The relationship between HMGB3, TGIF2, TLR3, and TGF- $\beta$ signaling was firstly disclosed, and we also further perfected the understanding of these molecules and their families. Targeting the TGIF2/HMGB3/TLR3 axis may be helpful for the diagnosis and treatment of ESCC. However, limitations in our study must also be discussed. Firstly, the number of ESCC patients in our cohort was relatively insufficient, which may explain TGIF2's insignificant role in the multicox regression analysis. Secondly, because some mice were in relatively poor condition before the xenograft experiment, we excluded them for ethical reasons. The number in different groups was not the same (4 or 5 as indicated). Thirdly, the reason why the combination of HMGB3 and TGIF2 could lead to the activation of TGF- $\beta$ signaling was not clearly verified. Consequently, further studies with a larger ESCC cohort and in-depth molecule mechanisms of TGIF2/HMGB3/TLR3 and in ESCC should be conducted in the future.

\section{Conclusion}

In conclusion, we firstly reported that TGIF2 could promote ESCC proliferation and metastasis via transcriptional regulation of HMGB. The inducing capability of TGIF2 is partly HMGB3-dependent. Additionally, TLR3 was verified as a novel binding partner of HMGB3. Combination of HMGB3 and TLR3 might be the reason for HMGB3 and TGIF2 to activate the Smad-dependent TGF- $\beta$ signaling.

Comprehension and investigation of the TGIF2/HMGB3/TLR3 axis in ESCC will provide novel perspective of the TGIF, HMGB and TLR family as well as the mechanism of ESCC progression. In addition, TGIF2/HMGB3/TLR3 axis may be promising target for treating ESCC.

\section{Abbreviations}

AJCC: American Joint Committee on Cancer; BMP: Bone morphogenetic proteins; ChIP: Chromatin Immunoprecipitation; EMT: Epithelial-mesenchymal transition; ESCC: Esophageal squamous cell carcinoma; H\&E: Hematoxylin and eosin; HADCs: histone deacetylases; CRC: Colorectal cancer; HCC: hepatocellular carcinoma; GC: gastric cancer; THCA: Thyroid carcinoma; HMGB: high mobility group box; HRP: horseradish peroxidase; IHC: immunohistochemistry; Co-IP: Co-immunoprecipitation; LPS: lipopolysaccharide; NC: Negative control; OS: Overall survival; PBS: Phosphate-buffered saline: qRT-PCR: Quantitative real-time polymerase chain reaction; PI: Propidium iodide; RAGE: advanced glycosylation end-product specific receptor; SPF: Specific pathogen-free; TALE: Three-amino acid loop extension; TCGA: The Cancer Genome Atlas; TGIF: TGF- $\beta$ interacting factor; TLR: Toll-like receptors.

\section{Declarations}




\section{Ethics approval and consent to participate}

The research was approved by the Xijing Hospital's Protection of Human Subjects Committee. We got the 21 pairs of ESCC tissue samples from patients in the Xijing Hospital, after signed informed consent obtained. Animal experiments were guaranteed in The Committee of the Use of Live Animals in Teaching and Research (CULATR) of the Fourth Military Medical University.

\section{Consent for publication}

All authors have agreed to publish this manuscript.

\section{Availability of data and materials}

The article and supplementary files included all the generated and analyzed data.

\section{Competing interests}

The authors declared no competing interests

\section{Funding}

This study was supported in part by grant from the National Natural Science Foundation of China (No. 82073210), grant from the Scientific Foundation of Shaanxi Province (No. S2019ZDLSF01-02-01; No. 2018SF-240) and grant from the National Clinical Research Center for Digestive Diseases (No. 2015BAI13B07)

\section{Author's contributions}

NLR, YWL, ZW and HL designed this experiment. WXQ, LYD AND WQ collected and prepared the related references. NLR and YWL drafted the manuscript. DLL, XCC, ZYJ and LJQ conceived the figures. ZQC, HL, and FDM checked and modified the manuscript. All authors read and approved the publish of final manuscript.

\section{Acknowledgements}

Not applicable

\section{Author's information}

1 State Key Laboratory of Cancer Biology and National Clinical Research Center for Digestive Diseases, Xijing Hospital of Digestive Diseases, Fourth Military Medical University, Xi'an, Shaanxi Province, 710032, China.

2 Department of Dermatology, Xijing Hospital, Fourth Military Medical University, Xi'an, Shaanxi Province, 710032 , China. 
394719 Military Hospital, Ji’an, Jiangxi Province, 343700, China.

\section{References}

1. Siegel RL, Miller KD, Jemal A. Cancer statistics, 2018. CA Cancer J Clin 2018; 68: 7-30.

2. Dinarvand $\mathrm{P}, \mathrm{Hassanian} \mathrm{SM}$, Qureshi $\mathrm{SH}$ et al. Polyphosphate amplifies proinflammatory responses of nuclear proteins through interaction with receptor for advanced glycation end products and P2Y1 purinergic receptor. Blood 2014; 123: 935-945.

3. Hanawa M, Suzuki S, Dobashi Y et al. EGFR protein overexpression and gene amplification in squamous cell carcinomas of the esophagus. Int J Cancer 2006; 118: 1173-1180.

4. Rustgi AK, El-Serag HB. Esophageal carcinoma. N Engl J Med 2014; 371: 2499-2509.

5. Pil PM, Lippard SJ. Specific binding of chromosomal protein HMG1 to DNA damaged by the anticancer drug cisplatin. Science 1992; 256: 234-237.

6. Sato $\mathrm{N}$, Koinuma J, Fujita $\mathrm{M}$ et al. Activation of WD repeat and high-mobility group box DNA binding protein 1 in pulmonary and esophageal carcinogenesis. Clin Cancer Res 2010; 16: 226-239.

7. Sun CX, Zhu F, Qi L. Demethylated miR-216a Regulates High Mobility Group Box 3 Promoting Growth of Esophageal Cancer Cells Through Wnt/ß-Catenin Pathway. Front Oncol 2021; 11: 622073.

8. Tang HR, Luo XQ, Xu G et al. High mobility group-box 3 overexpression is associated with poor prognosis of resected gastric adenocarcinoma. World J Gastroenterol 2012; 18: 7319-7326.

9. Zheng W, Yang J, Dong Z et al. High mobility group box 3 as an emerging biomarker in diagnosis and prognosis of hepatocellular carcinoma. Cancer Manag Res 2018; 10: 5979-5989.

10. Gao J, Zou Z, Gao J et al. Increased expression of HMGB3: a novel independent prognostic marker of worse outcome in patients with esophageal squamous cell carcinoma. Int J Clin Exp Pathol 2015; 8: 345-352.

11. Yamada $Y$, Nishikawa R, Kato $M$ et al. Regulation of HMGB3 by antitumor miR-205-5p inhibits cancer cell aggressiveness and is involved in prostate cancer pathogenesis. J Hum Genet 2018; 63: 195205.

12. Wang LK, Xie XN, Song XH et al. Upregulation of miR-200b Inhibits Hepatocellular Carcinoma Cell Proliferation and Migration by Targeting HMGB3 Protein. Technol Cancer Res Treat 2018; 17: 1533033818806475.

13. Zhang C, Samanta D, Lu H et al. Hypoxia induces the breast cancer stem cell phenotype by HIFdependent and ALKBH5-mediated m(6)A-demethylation of NANOG mRNA. Proc Natl Acad Sci U S A 2016; 113: E2047-2056.

14. Mukherjee A, Huynh V, Gaines K et al. Targeting the High-Mobility Group Box 3 Protein Sensitizes Chemoresistant Ovarian Cancer Cells to Cisplatin. Cancer Res 2019; 79: 3185-3191.

15. Saghafinia S, Homicsko K, Di Domenico A et al. Cancer cells retrace a stepwise differentiation program during malignant progression. Cancer Discov 2021. 
16. Wang J, Sheng Z, Cai Y. Effects of microRNA-513b on cell proliferation, apoptosis, invasion, and migration by targeting HMGB3 through regulation of mTOR signaling pathway in non-small-cell lung cancer. J Cell Physiol 2019; 234: 10934-10941.

17. Zhang Z, Chang Y, Zhang J et al. HMGB3 promotes growth and migration in colorectal cancer by regulating WNT/ß-catenin pathway. PLoS One 2017; 12: e0179741.

18. Zhang $C$, Samanta $D$, Lu $H$ et al. Hypoxia induces the breast cancer stem cell phenotype by HIFdependent and ALKBH5-mediated mヌA-demethylation of NANOG mRNA. Proc Natl Acad Sci U S A 2016; 113: E2047-2056.

19. Li N, Jiang D, He Q et al. microRNA-181c-5p promotes the formation of insulin-producing cells from human induced pluripotent stem cells by targeting smad7 and TGIF2. Cell Death Dis 2020; 11: 462.

20. Spagnoli FM, Brivanlou AH. The Gata5 target, TGIF2, defines the pancreatic region by modulating BMP signals within the endoderm. Development 2008; 135: 451-461.

21. Haellman V, Saxena P, Jiang Y, Fussenegger M. Rational design and optimization of synthetic gene switches for controlling cell-fate decisions in pluripotent stem cells. Metab Eng 2021; 65: 99-110.

22. Hamabe $\mathrm{A}$, Konno $\mathrm{M}$, Tanuma $\mathrm{N}$ et al. Role of pyruvate kinase $\mathrm{M} 2$ in transcriptional regulation leading to epithelial-mesenchymal transition. Proc Natl Acad Sci U S A 2014; 111: 15526-15531.

23. Wotton D, Lo RS, Lee S, Massagué J. A Smad transcriptional corepressor. Cell 1999; 97: 29-39.

24. Du R, Shen W, Liu Y et al. TGIF2 promotes the progression of lung adenocarcinoma by bridging EGFR/RAS/ERK signaling to cancer cell stemness. Signal Transduct Target Ther 2019; 4: 60.

25. Tsuzuki S, Seto M. TEL (ETV6)-AML1 (RUNX1) initiates self-renewing fetal pro-B cells in association with a transcriptional program shared with embryonic stem cells in mice. Stem Cells 2013; 31: 236247.

26. Derynck R, Zhang YE. Smad-dependent and Smad-independent pathways in TGF-beta family signalling. Nature 2003; 425: 577-584.

27. Massagué J. How cells read TGF-beta signals. Nat Rev Mol Cell Biol 2000; 1: 169-178.

28. Massagué J. TGFbeta in Cancer. Cell 2008; 134: 215-230.

29. Natsugoe $S$, Xiangming $C$, Matsumoto $M$ et al. Smad4 and transforming growth factor beta 1 expression in patients with squamous cell carcinoma of the esophagus. Clin Cancer Res 2002; 8 : 1838-1842.

30. Natsuizaka M, Whelan KA, Kagawa S et al. Interplay between Notch1 and Notch3 promotes EMT and tumor initiation in squamous cell carcinoma. Nat Commun 2017; 8: 1758.

31. Noma K, Smalley KS, Lioni M et al. The essential role of fibroblasts in esophageal squamous cell carcinoma-induced angiogenesis. Gastroenterology 2008; 134: 1981-1993.

32. Liu A, Zhu J, Wu G et al. Antagonizing miR-455-3p inhibits chemoresistance and aggressiveness in esophageal squamous cell carcinoma. Mol Cancer 2017; 16: 106.

33. Zhao X, Liu S, Chen X et al. L1CAM overexpression promotes tumor progression through recruitment of regulatory T cells in esophageal carcinoma. Cancer Biol Med 2021. 
34. Lin M, Yiu WH, Li RX et al. The TLR4 antagonist CRX-526 protects against advanced diabetic nephropathy. Kidney Int 2013; 83: 887-900.

35. Li C, Li H, Jiang K et al. TLR4 signaling pathway in mouse Lewis lung cancer cells promotes the expression of TGF- $\beta 1$ and IL-10 and tumor cells migration. Biomed Mater Eng 2014; 24: 869-875.

36. Cerdá-Esteban N, Naumann H, Ruzittu S et al. Stepwise reprogramming of liver cells to a pancreas progenitor state by the transcriptional regulator Tgif2. Nat Commun 2017; 8: 14127.

37. Agresti A, Bianchi ME. HMGB proteins and gene expression. Curr Opin Genet Dev 2003; 13: 170-178.

38. Xie X, Pan J, Han X, Chen W. Downregulation of microRNA-532-5p promotes the proliferation and invasion of bladder cancer cells through promotion of HMGB3/Wnt/ $\beta$-catenin signaling. Chem Biol Interact 2019; 300: 73-81.

39. Chen X, Zeng L. Ginkgo biloba extract 761 enhances 5 -fluorouracil chemosensitivity in colorectal cancer cells through regulation of high mobility group-box 3 expression. Am J Transl Res 2018; 10: 1773-1783.

40. Dey A, Kang X, Qiu J et al. Anti-Inflammatory Small Molecules To Treat Seizures and Epilepsy: From Bench to Bedside. Trends Pharmacol Sci 2016; 37: 463-484.

41. Thomas DA, Massagué J. TGF-beta directly targets cytotoxic T cell functions during tumor evasion of immune surveillance. Cancer Cell 2005; 8: 369-380.

42. De Wever O, Mareel M. Role of tissue stroma in cancer cell invasion. J Pathol 2003; 200: 429-447.

43. Kingsley LA, Fournier PG, Chirgwin JM, Guise TA. Molecular biology of bone metastasis. Mol Cancer Ther 2007; 6: 2609-2617.

44. Lawrimore CJ, Crews FT. Ethanol, TLR3, and TLR4 Agonists Have Unique Innate Immune Responses in Neuron-Like SH-SY5Y and Microglia-Like BV2. Alcohol Clin Exp Res 2017; 41: 939-954.

45. Boes M, Meyer-Wentrup F. TLR3 triggering regulates PD-L1 (CD274) expression in human neuroblastoma cells. Cancer Lett 2015; 361: 49-56.

46. Natarajan S, Ranganathan M. Toll-like receptor (TLR) gene expression and immunostimulatory effect of $\mathrm{CpG}$ oligonucleotides in hormone receptor positive cell line T47D and triple negative breast cancer cell line MDA-MB-468. Immunopharmacol Immunotoxicol 2020; 42: 408-415.

47. Shinde-Jadhav S, Mansure JJ, Rayes RF et al. Role of neutrophil extracellular traps in radiation resistance of invasive bladder cancer. Nat Commun 2021; 12: 2776.

\section{Tables}

Table1 Correlation between HMGB3, TGIF2 expression and Clinicopathological characteristics of ESCC in an independent cohort of human ESCC tissue 
Clinicopathological variables
Tumor HMGB3 expression

$\begin{array}{ll}\text { Low } & \text { High } \\ \text { expression } & \text { expression } \\ n=55 & n=57\end{array}$

P- $\quad$ Tumor TGIF2 expression value $\begin{array}{ll}\text { Low } & \text { High } \\ \text { expression } & \text { expression } \\ n=56 & \end{array}$ $\mathrm{n}=56$
Age

$\leq 65$

$>65$

Gender

female

male

Pathological grade

$$
\text { प }
$$

口

[

Tumor size $(\mathrm{cm})$

$\leq 5$

$>5$

Tumor invasion

$\mathrm{T} 1$

T2

T3

$\mathrm{T} 4$

Lymph node

metastasis

Absent

Present

American Joint

Committee on Cancer

(AJCC) stage
0.26

24

33

26

29

16

19

37

10
47

47

0.046

0.105

Pvalue

0.663

$\begin{array}{ll}22 & 13 \\ 33 & 43\end{array}$

13

43

$15 \quad 31$

$41 \quad 25$

0.369

0.006

$\begin{array}{llll}2 & 5 & 1 & 6 \\ 40 & 42 & 37 & 44 \\ 14 & 10 & 18 & 6\end{array}$

0.161

0.079

$\begin{array}{llll}40 & 34 & 40 & 33 \\ 13 & 20 & 12 & 21\end{array}$

0.05

0.067

$\begin{array}{llll}4 & 1 & 2 & 3 \\ 13 & 5 & 14 & 4 \\ 36 & 47 & 38 & 44 \\ 0 & 1 & 0 & 1\end{array}$

0.074

0.705

$\begin{array}{lllll}\text { Stage } \ & 4 & 1 & 2 & 3 \\ \text { Stage } \ & 30 & 18 & 29 & 18 \\ & & \text { Page 20/33 } & & \end{array}$


Table2 Univariate and multivariate analysis of the factors associated with OS in ESCC patients

Variables $(\mathrm{N}=112)$

\begin{tabular}{|c|c|c|c|c|c|c|}
\hline \multirow[t]{3}{*}{ Variables $(\mathrm{N}=112)$} & \multicolumn{6}{|c|}{ Overall Survival } \\
\hline & \multicolumn{3}{|c|}{ Univariate analysis } & \multicolumn{3}{|c|}{ multivariate analysis } \\
\hline & $\mathrm{HR}$ & $95 \% \mathrm{Cl}$ & $\begin{array}{l}\mathrm{P}- \\
\text { value }\end{array}$ & $\mathrm{HR}$ & $\begin{array}{l}95 \% \\
\mathrm{Cl}\end{array}$ & $\begin{array}{l}\mathrm{P}- \\
\text { value }\end{array}$ \\
\hline Age $(\leq 65$ versus $>65)$ & 2.177 & $\begin{array}{l}1.295- \\
3.658\end{array}$ & 0.003 & 1.502 & $\begin{array}{l}0.909- \\
2.482\end{array}$ & 0.113 \\
\hline Gender (male versus female) & 1.011 & $\begin{array}{l}0.671- \\
1.524\end{array}$ & 0.958 & 2.477 & $\begin{array}{l}1.305- \\
4.701\end{array}$ & 0.006 \\
\hline Pathological grade (I/II versus III) & 0.743 & $\begin{array}{l}0.444- \\
1.243\end{array}$ & 0.258 & 0.965 & $\begin{array}{l}0.546- \\
1.707\end{array}$ & 0.903 \\
\hline Tumor size $(\leq 5$ versus $>5$ ) & 1.55 & $\begin{array}{l}0.988- \\
2.432\end{array}$ & 0.057 & 1.453 & $\begin{array}{l}0.878- \\
2.404\end{array}$ & 0.146 \\
\hline Tumor invasion ( $\mathrm{T} 1-\mathrm{T} 2$ versus $\mathrm{T} 3-\mathrm{T} 4)$ & 2.661 & $\begin{array}{l}1.491- \\
4.750\end{array}$ & 0.001 & 2.09 & $\begin{array}{l}1.009- \\
4.331\end{array}$ & 0.047 \\
\hline $\begin{array}{l}\text { Lymph node metastasis (absent versus } \\
\text { present) }\end{array}$ & 1.8 & $\begin{array}{l}1.184- \\
2.737\end{array}$ & 0.006 & 1.184 & $\begin{array}{l}0.446- \\
3.143\end{array}$ & 0.735 \\
\hline $\begin{array}{l}\text { American Joint Committee on Cancer } \\
\text { (AJCC) stage (I-II versus III-IV) }\end{array}$ & 3.163 & $\begin{array}{l}2.004- \\
4.991\end{array}$ & $<0.001$ & 1.22 & $\begin{array}{l}0.395- \\
3.763\end{array}$ & 0.729 \\
\hline HMGB3 expression (low versus high) & 4.914 & $\begin{array}{l}3.135- \\
7.705\end{array}$ & $<0.001$ & 3.583 & $\begin{array}{l}2.029- \\
6.334\end{array}$ & $<0.001$ \\
\hline TGIF2 expression (low versus high) & 2.325 & $\begin{array}{l}1.525- \\
3.543\end{array}$ & $<0.001$ & 1.617 & $\begin{array}{l}0.946- \\
2.764\end{array}$ & 0.079 \\
\hline
\end{tabular}

\section{Figures}



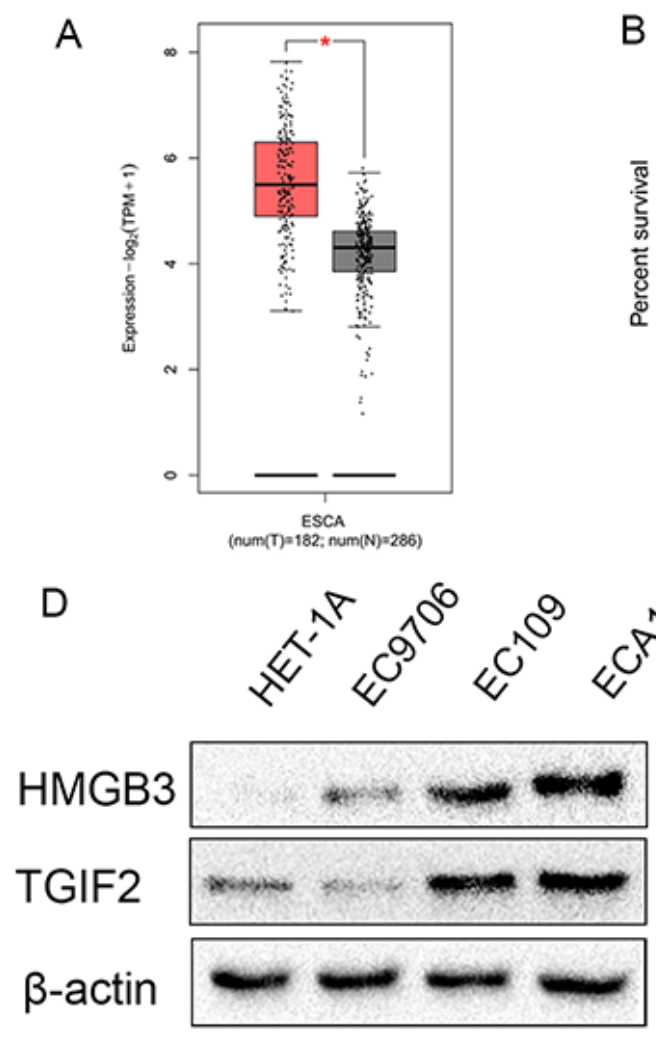

G

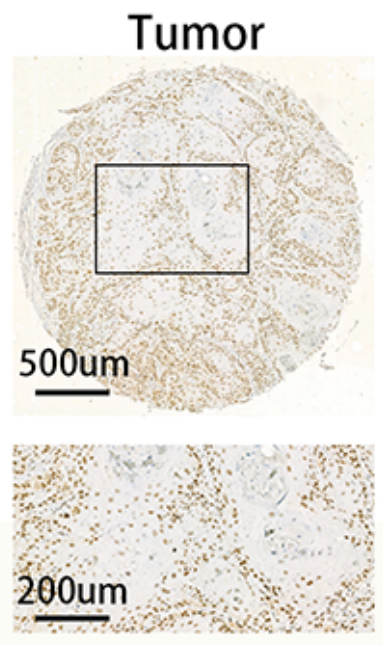

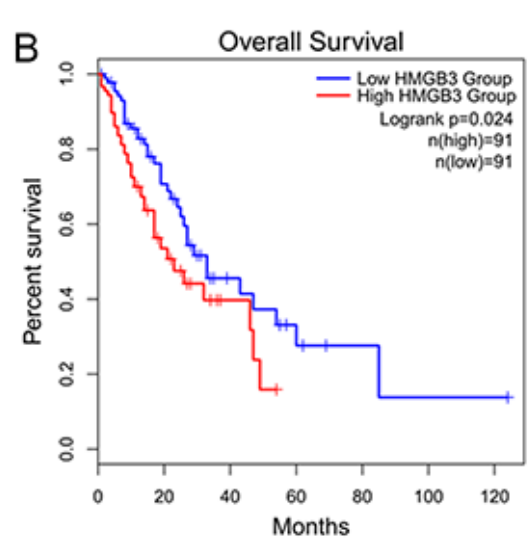

C

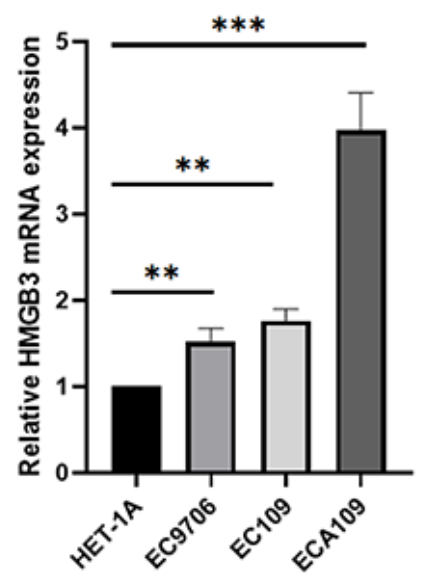

$\mathrm{F}$

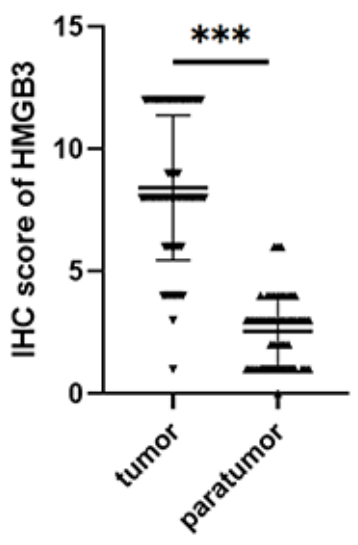

$\mathrm{H}$
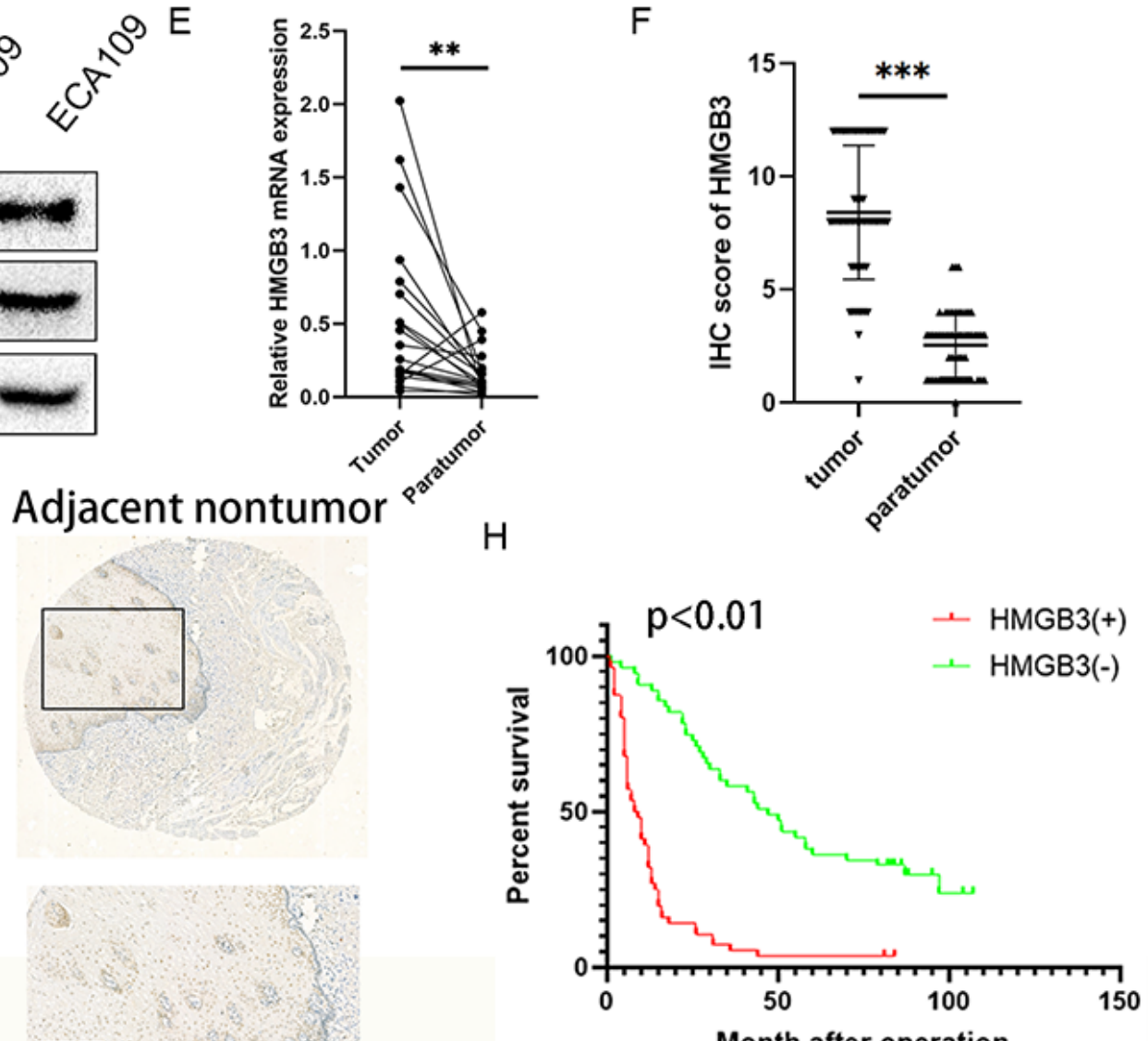

Month after operation

\section{Figure 1}

Overexpression of HMGB3 in ESCC positively correlates with poor prognosis. A. Data extracted from TCGA datasets illustrates the expression of HMGB3 mRNA level in ESCC patients $(N=182)$ and normal ones $(\mathrm{N}=286)$. The box-and-whisker plots indicate the medians (horizontal lines), interquartile ranges (boxes), minimum values, and maximum values (whiskers) of the data. B. Kaplan-Meier analysis illustrates the relationship between HMGB3 mRNA expression and OS for the patients in the TCGA datasets. Red line indicates the group with higher HMGB3 expression, while blue line indicates patients with lower HMGB3 expression. C. Relative HMGB3 mRNA expression level in ESCC cell lines analyzed by qRT-PCR. D. Relative HMGB3 and TGIF2 protein expression level in ESCC cell lines analyzed by WB. E. qRT-PCR analysis shows the HMGB3 expression level in 21 pairs ESCC tissues and corresponding 
adjacent normal tissues. F. Semiquantitative analysis was employed to evaluate the scores of the expression of HMGB3 evaluated in the ESCC tissue microarray ( $N=57)$. G. Representative IHC image for HMGB3 staining in the ESCC tissue microarray. H. Kaplan-Meier analysis illustrates the relationship between HMGB3 mRNA expression and OS for the patients in the ESCC tissue microarray $(N=112)$. All the data are shown as the mean \pm SD. ${ }^{* * *} P<0.001,{ }^{*} P<0.01$ and $* P<0.05$.
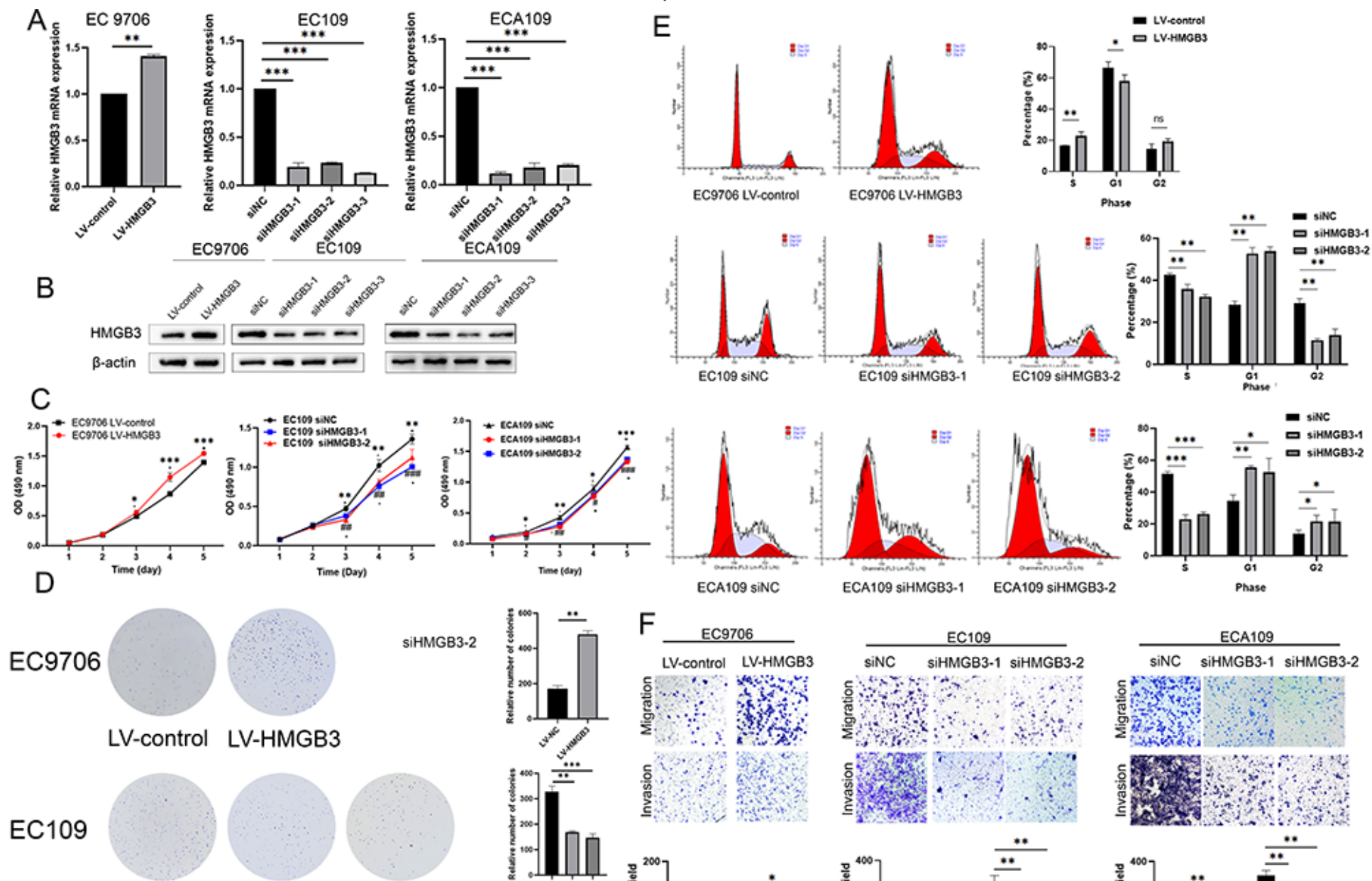

siNC siHMGB3-1 siHMGB3-2
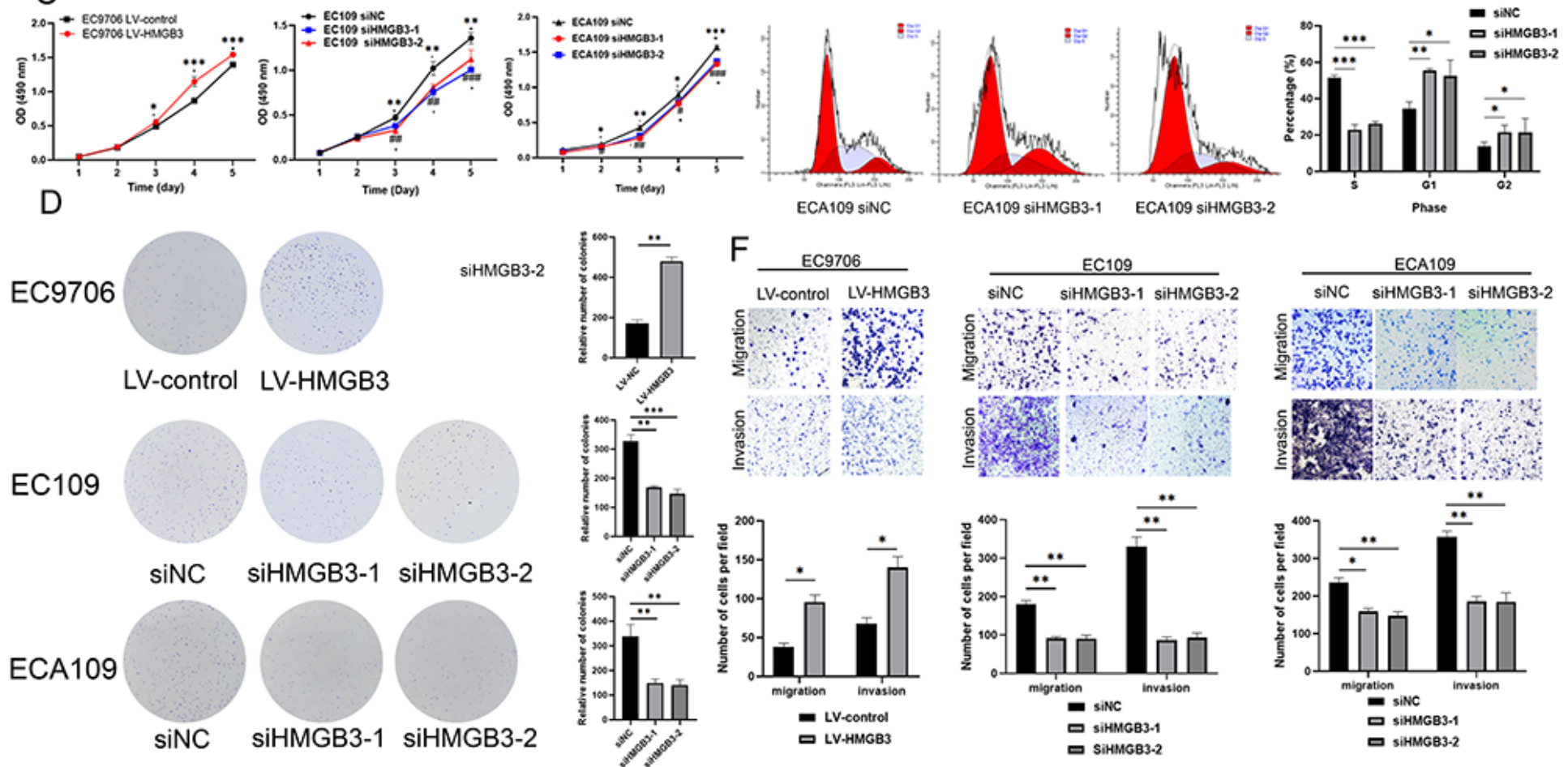

\section{Figure 2}

HMGB3 promotes ESCC cell proliferation, migration, and invasion in vitro A. Relative HMGB3 mRNA expression level in EC9706, EC109 and ECA109 cells after lentivirus infection or si-RNA transfection, analyzed by qRT-PCR. B. Relative HMGB3 protein expression level in EC9706, EC109 and ECA109 cells after lentivirus infection or si-RNA transfection, analyzed by WB. C. CCK-8 assay illustrates the proliferative capacity of ESCC cell after the manipulation of HMGB3 expression, and the OD was observed every day in 5 days. D. Colony formation illustrates the proliferative capacity of ESCC cell after the manipulation of HMGB3 expression (left), and the number of colonies in indicated group were counted (right) (* means siNC vs siHMGB3-1, while \# means siNC vs siHMGB3-2 in EC109 and ECA109). 
E. Flow cytometry analysis assesses the effect of HMGB3 on cell cycle (left), and the percentage of S, G1, G2 phase was calculated (right). F. Transwell analysis illustrates the migrative and invasive capability of ESCC cell after the manipulation of HMGB3 expression (up), and the number of migrated cells were calculated (down). All the data are shown as the mean $\pm S D$. ${ }^{\star \star *} P<0.001, * \star P<0.01$ and $* P<0.05$. \#\#\# $P<0.001, \# \#<0.01$ and $\# P<0.05$.
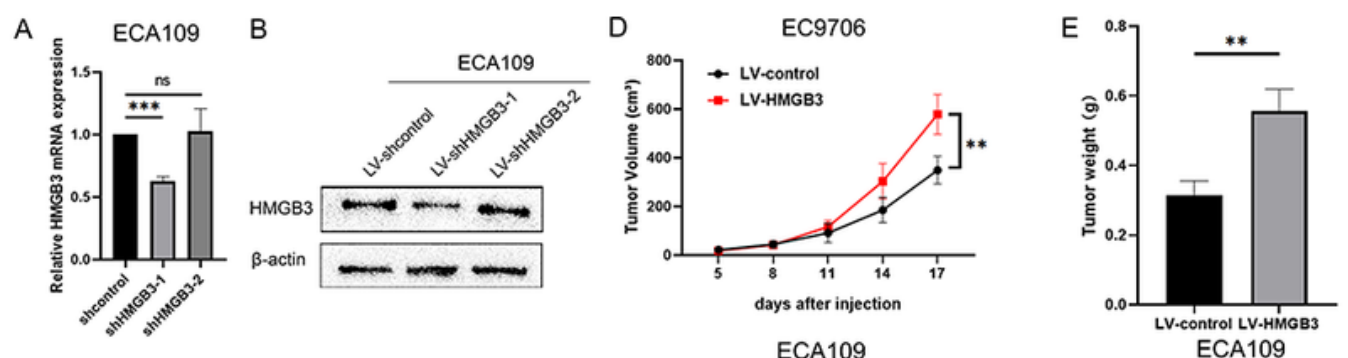

C
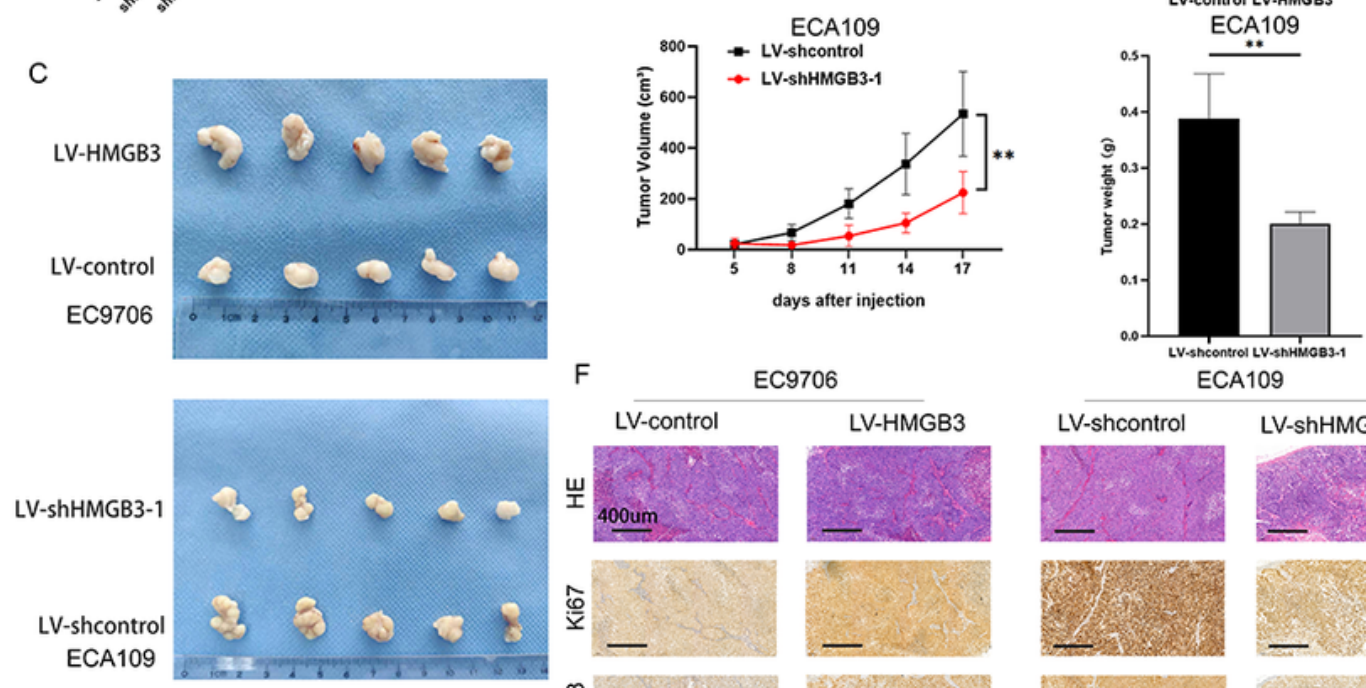

F LV-control
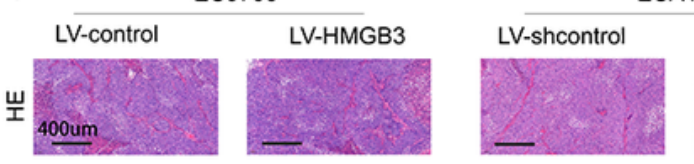

LV-shHMGB3-1 ECA109
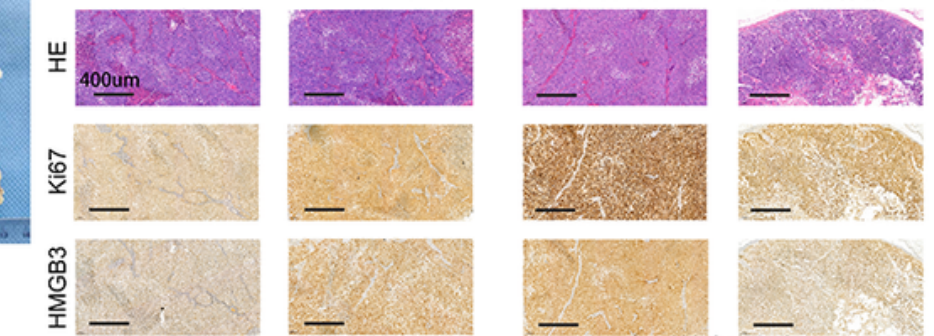

G
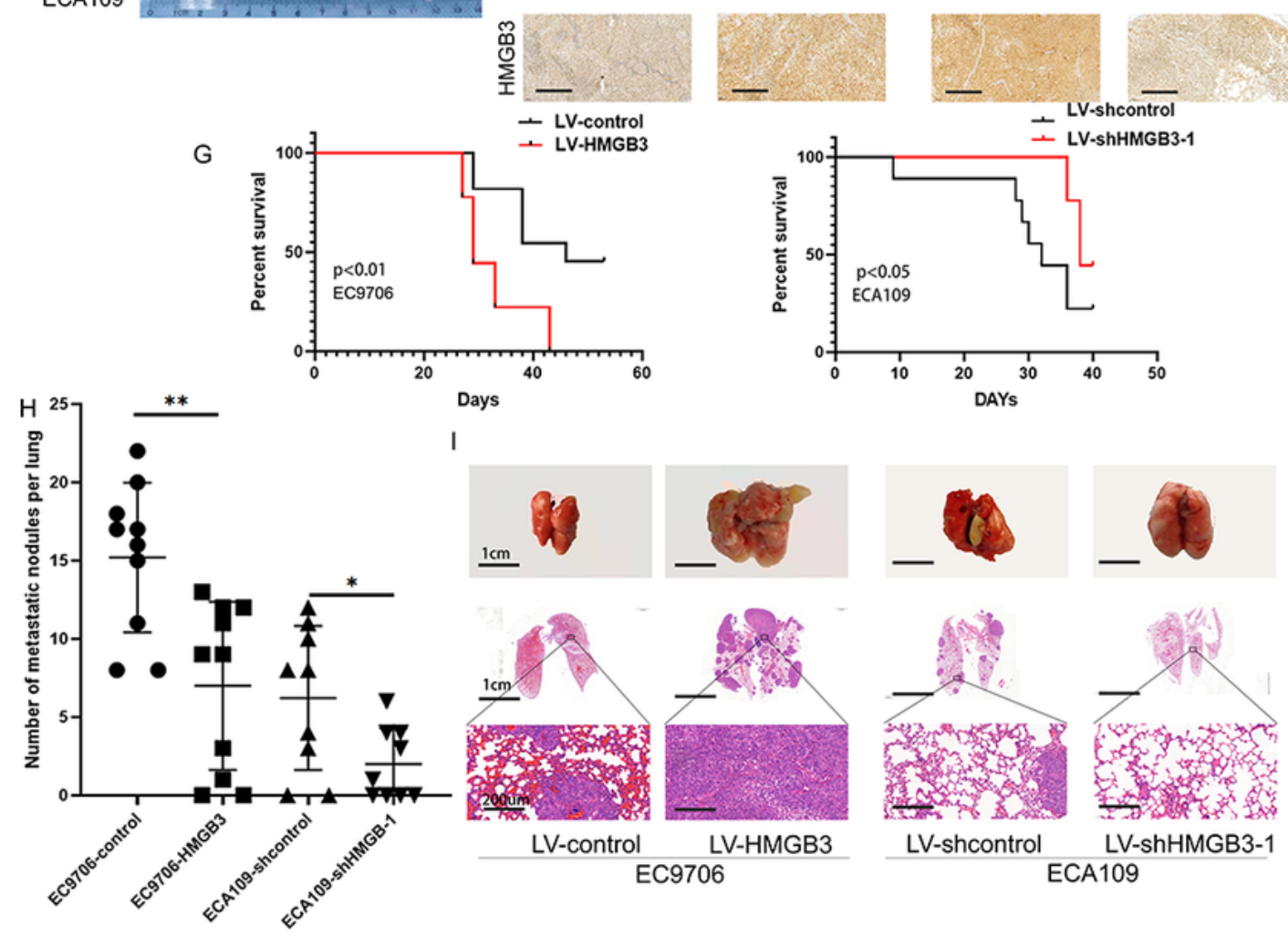

Figure 3 
HMGB3 promotes ESCC cell proliferation, and migration in vivo A. Relative HMGB3 mRNA expression level in ECA109 after lentivirus infection, analyzed by qRT-PCR. B. Relative HMGB3 protein expression level in ECA109 after lentivirus infection, analyzed by WB. C-F. Nude mice were classified into four groups ( $N=5$ mice per group) and subcutaneously injected with indicated cells. Representative images of the xenograft mouse tumors were presented in $\mathrm{C}$. The data of tumor volumes and tumor weight of the xenograft mouse tumors was shown in D, E. Representative images of the HE staining, IHC staining for HMGB3 and Ki67 in the xenograft mouse tumors were presented in F. G-I. Nude mice were classified into four groups ( $N=10$ mice per group) and injected with indicated cells by tail vein. $G$. OS of the four groups of nude mice. H. Calculated number of metastatic nodules in lung of the different groups. I.

Representative images of H\&E staining of the lung samples from the indicated groups. All the data are shown as the mean \pm SD. ${ }^{\star \star *} P<0.001, * * P<0.01$, and $* P<0.05$. 
A UCSC Genome Browser on Human Dec. 2013 (GRCh38ng s8) Assembly

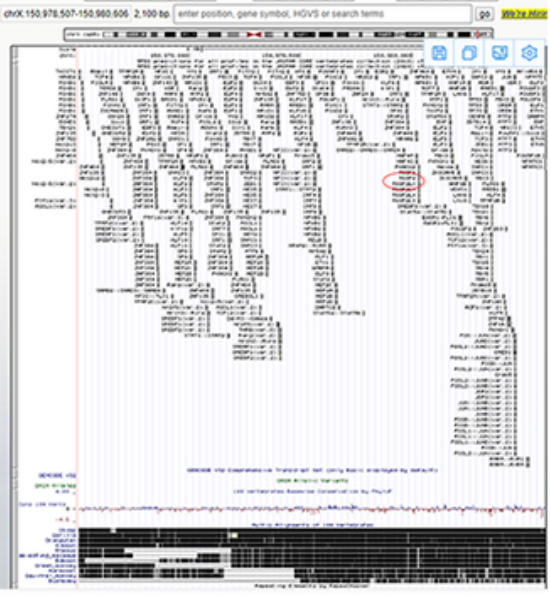

C

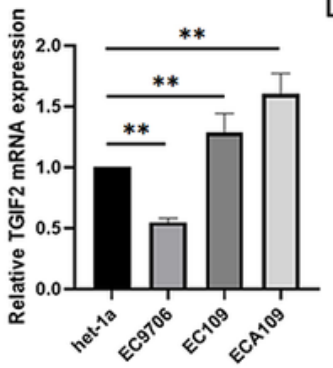

D

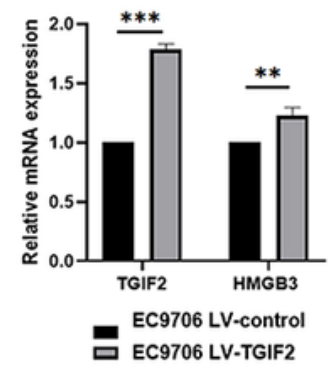

E
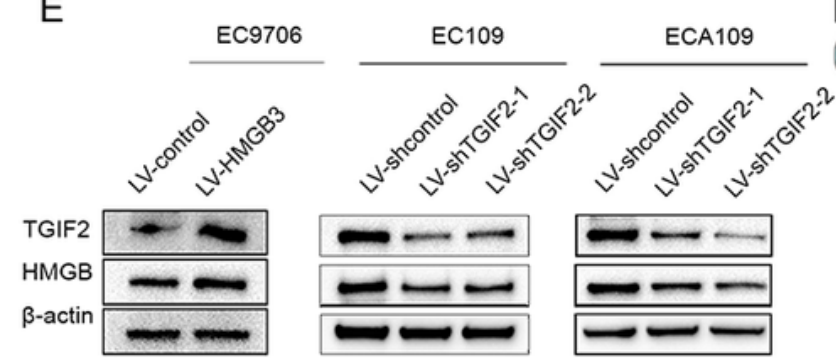

F 口 EC109 LV-ShTGIF2-2
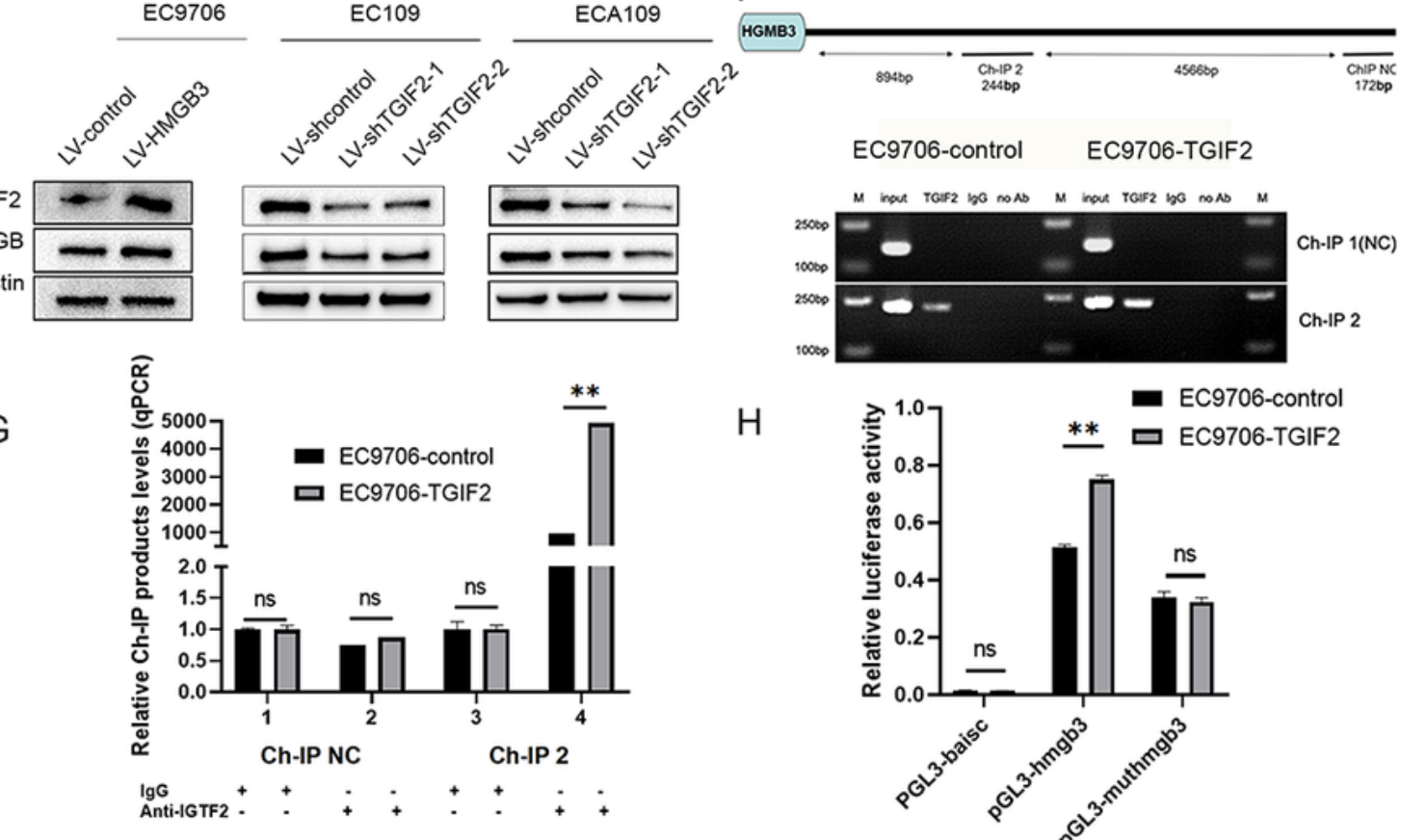

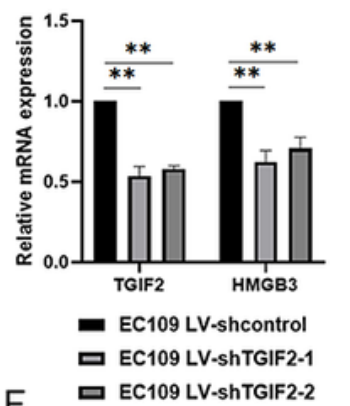

B

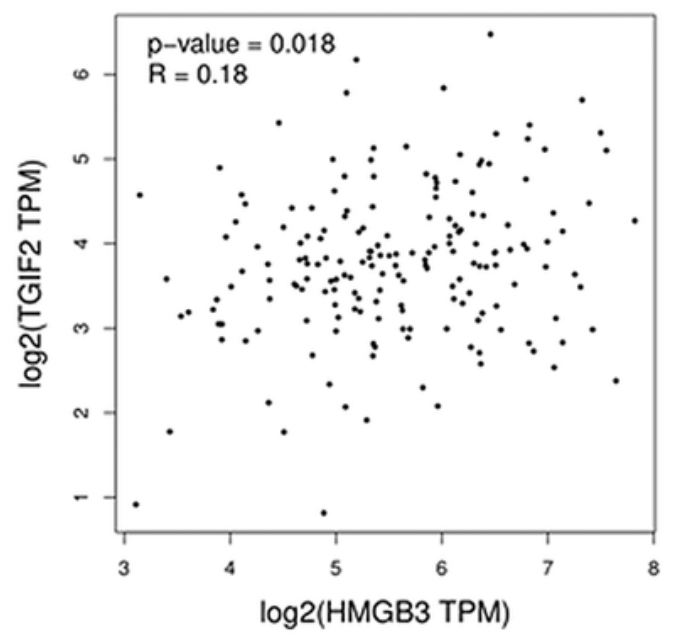

log2(HMGB3 TPM)

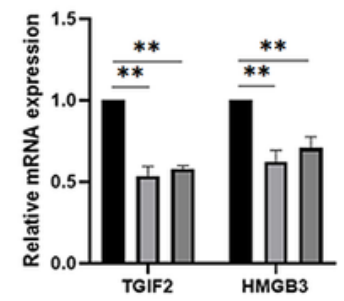

- EC109 LV-shcontrol

ㅁ EC109 LV-shTGIF2-1

口 EC109 LV-shTGIF2-2
G

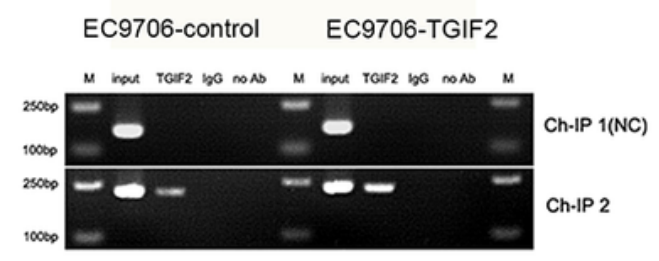

$\mathrm{H}$

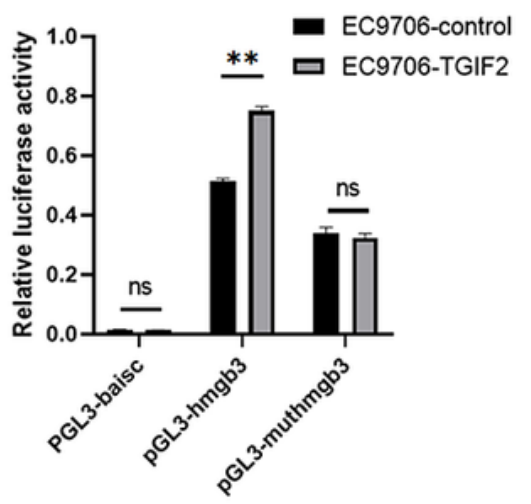

Figure 4

HMGB3 is transcriptionally regulated by TGIF2 A. Prediction of HMGB3's transcriptional factor by JAPAR database. B. Correlation analysis between the expression of TGIF2 and HMGB3 in TCGA database. C. Relative TGIF2 mRNA expression level in ESCC cell lines and normal het-1A esophageal cell, analyzed by qRT-PCR. D. Relative TGIF2 and HMGB3 mRNA expression level in EC9706, EC109 and ECA109 cells after lentivirus infection, analyzed by qRT-PCR. E. Relative TGIF2 and HMGB3 protein expression level in 
EC9706, EC109 and ECA109 cells after lentivirus infection, analyzed by WB. F, G. ChIP assays demonstrates that TGIF2 directly bind to the HMGB3 promoter and identifies the binding site between -1138 bp to -894 bp. H. EC9706-control and EC9706-TGIF2 cells were infected with serially truncated and mutated HMGB3 promoter which was cloned into pGL3-luciferase reporter plasmids. Relative luciferase activity was performed afterwards. All the data are shown as the mean $\pm S D$. ${ }^{\star \star *} P<0.001,{ }^{*} P<0.01$ and $* \mathrm{P}<0.05$.

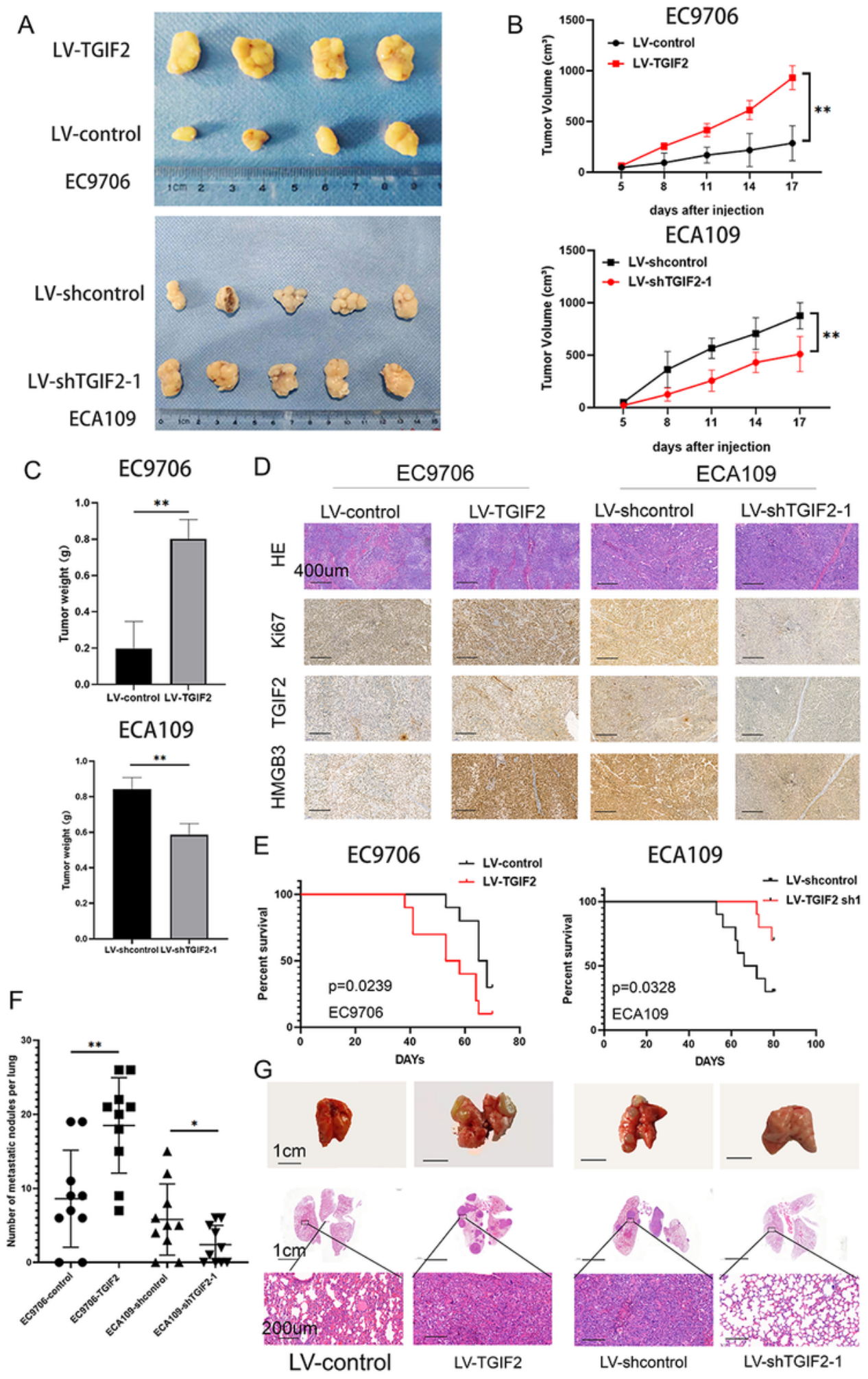

Figure 5 
TGIF2 promotes ESCC cell proliferation, and migration in vivo A-D. Nude mice were classified into four groups ( $\mathrm{N}=4$ or 5 mice per group) and subcutaneously injected with indicated cells. Representative images of the xenograft mouse tumors were presented in $A$. The data of tumor volumes and tumor weight of the xenograft mouse tumors was shown in B, C. Representative images of the HE staining, IHC staining for TGIF2, HMGB3 and Ki67 in the xenograft mouse tumors were presented in D. E-G. Nude mice were classified into four groups ( $N=10$ mice per group) and injected with indicated cells by tail vein. $E$. OS of the four groups of nude mice. F. Calculated number of metastatic nodules in lung of the different groups. G. Representative images of H\&E staining of the lung samples from the indicated groups. All the data are shown as the mean $\pm S D$. ${ }^{\star * \star} P<0.001,{ }^{*} \mathrm{P}<0.01$, and $* \mathrm{P}<0.05$. 
A
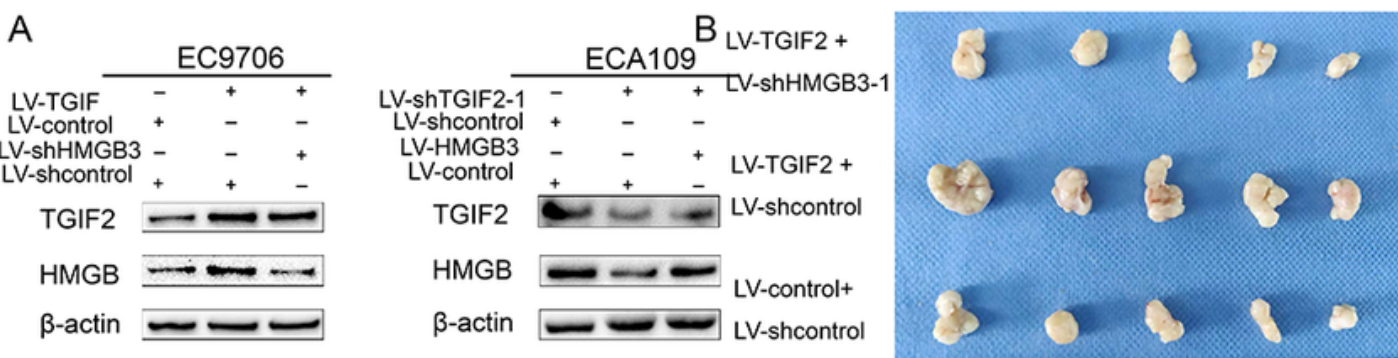

E

EC9706

EC9706

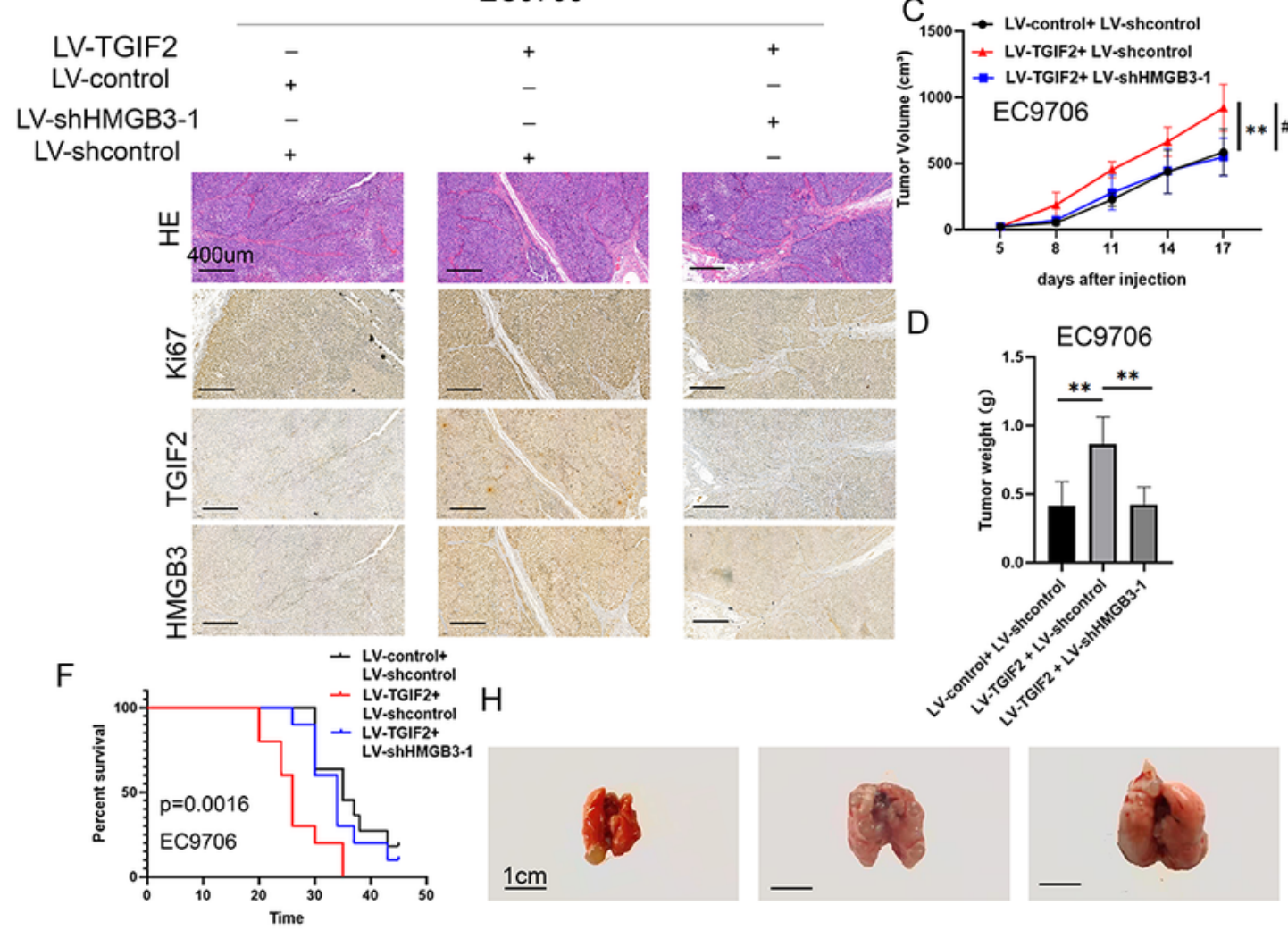

G
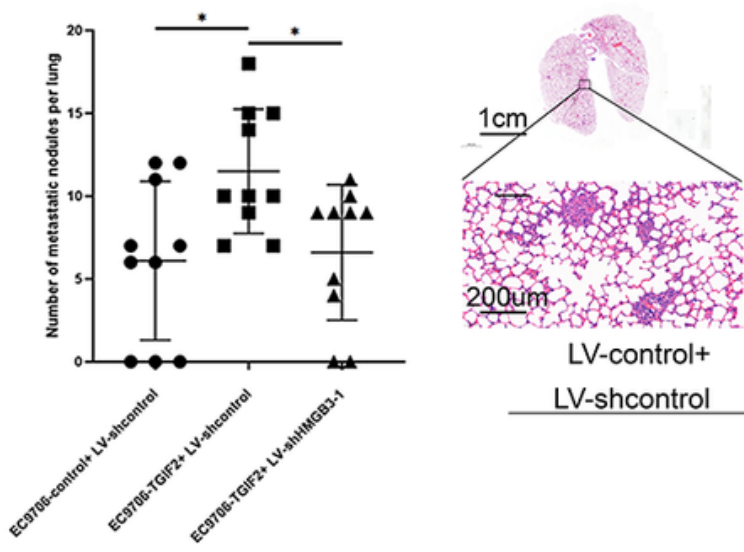

20 um

LV-control+

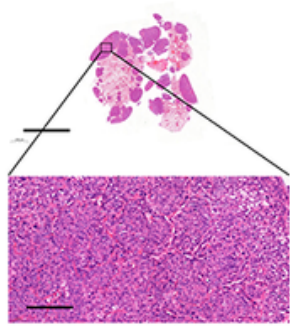

LV-TGIF2+

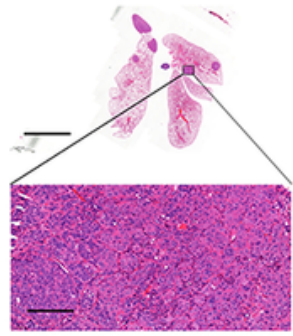

LV-shcontrol

LV-shcontrol

LV-TGIF2+

EC9706

LV-shHMGB3-1

\section{Figure 6}

HMGB3 is essential for TGIF2-mediated ESCC cell proliferation and metastasis. A. Relative HMGB3 and TGIF2 protein expression level in EC9706, and ECA109 cells after lentivirus infection, analyzed by WB. BE. Nude mice were classified into three groups ( $N=5$ mice per group) and subcutaneously injected with indicated cells. Representative images of the xenograft mouse tumors were presented in B. The data of tumor volumes and tumor weight of the xenograft mouse tumors was shown in C, D. Representative 
images of the HE staining, IHC staining for HMGB3 and Ki67 in the xenograft mouse tumors were presented in $\mathrm{E}$. F-H. Nude mice were classified into four groups ( $\mathrm{N}=10$ mice per group) and injected with indicated cells by tail vein. F. OS of the three groups of nude mice. G. Calculated number of metastatic nodules in lung of the different groups. $\mathrm{H}$. Representative images of H\&E staining of the lung samples from the indicated groups. All the data are shown as the mean $\pm S D$. ${ }^{\star \star *} P<0.001,{ }^{*} P<0.01$ and ${ }^{*} P<0.05$. $\# \# \# P<0.001$, \#\#P< 0.01 and \#P $<0.05$.

A
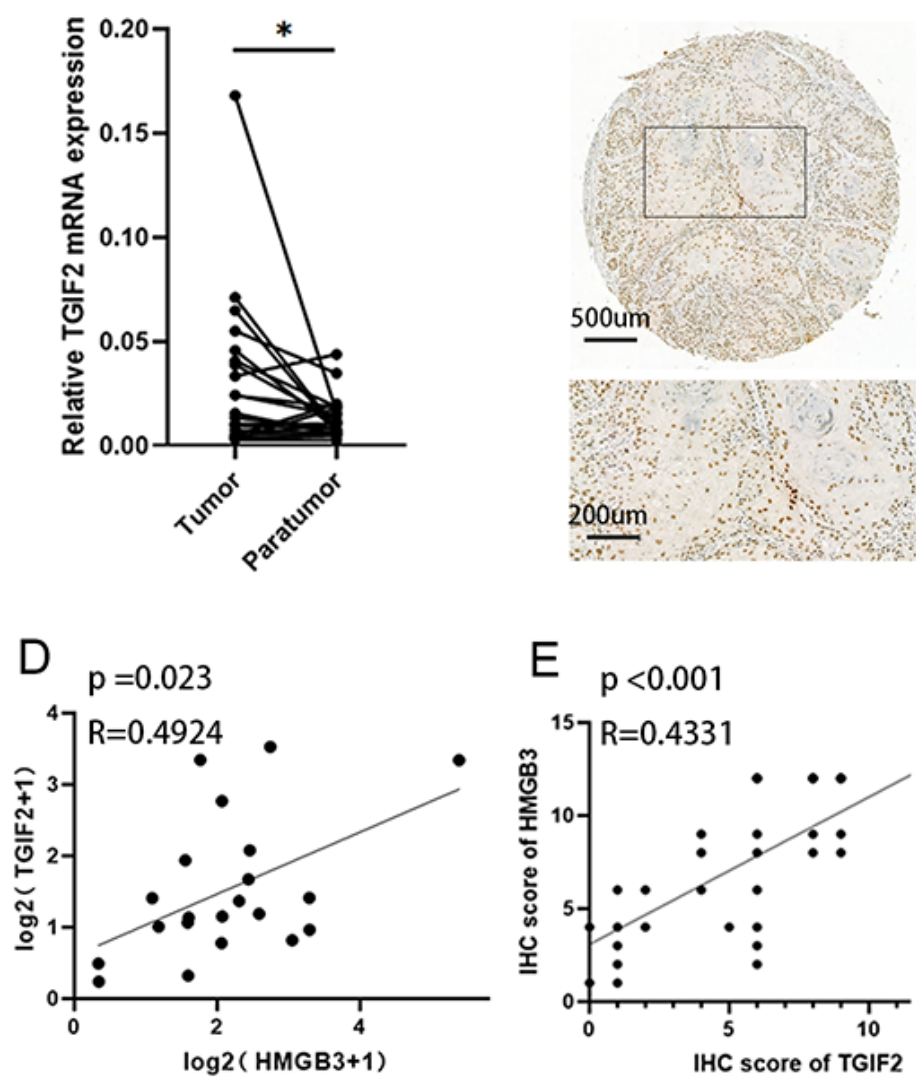

G

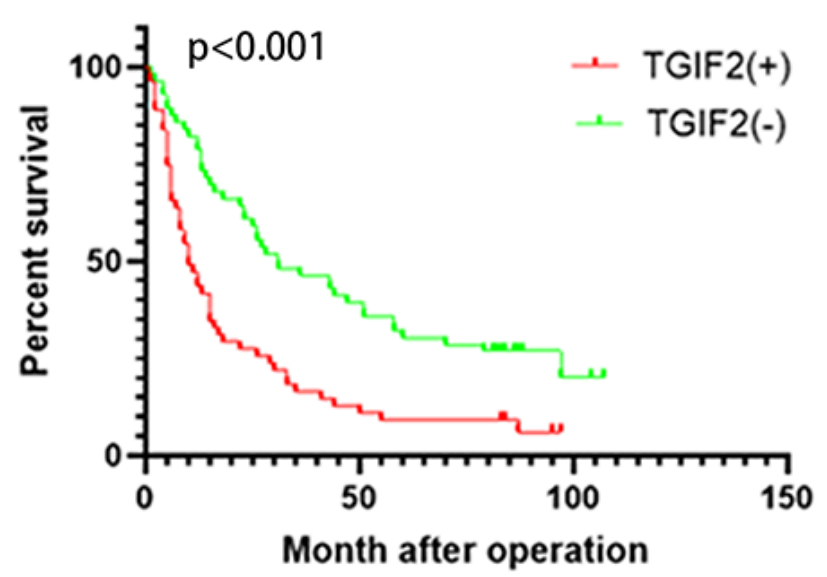

E $p<0.001$

B Tumor

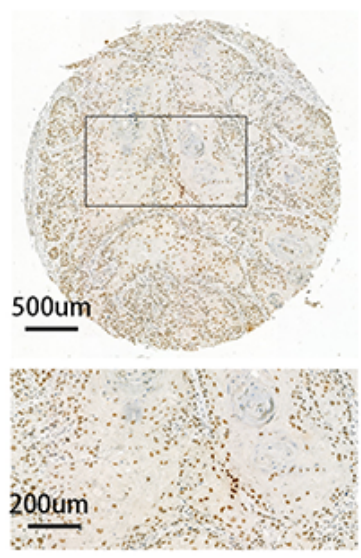

IHC score of TGIF2
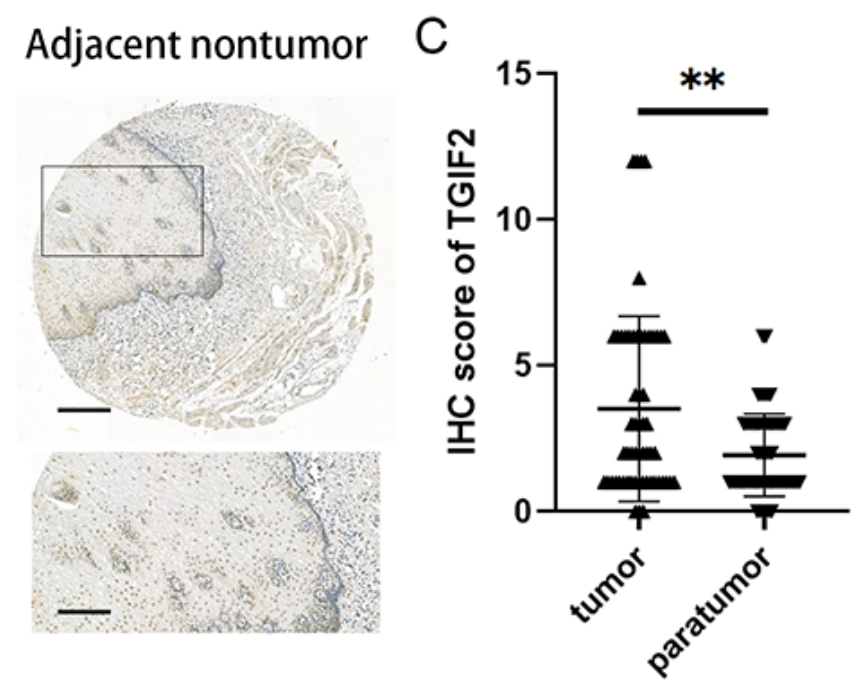

F

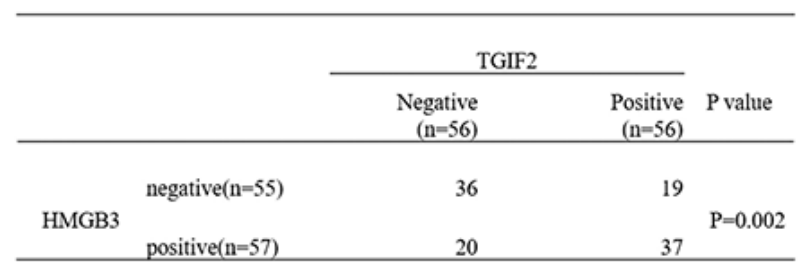

$\mathrm{H}$

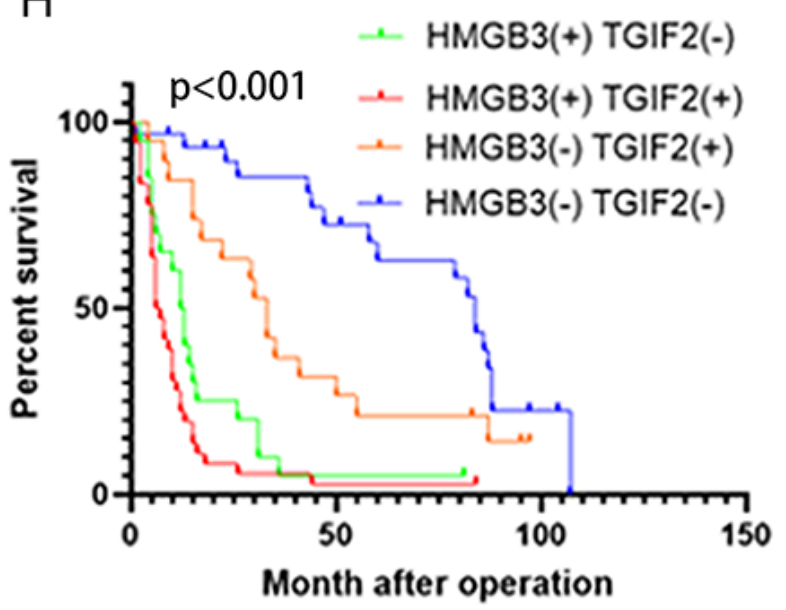

Figure 7 
Clinical significance and correlation between HMGB3 and TGIF2 A. qRT-PCR analysis shows the HMGB3 expression level in 21 pairs ESCC tissues and corresponding adjacent normal tissues. B. Representative IHC image for TGIF2 staining in the ESCC tissue microarray. C. Semiquantitative analysis was employed to evaluate the scores of the expression of HMGB3 evaluated in the ESCC tissue microarray $(\mathrm{N}=57$ pairs). D. Correlation analysis of the mRNA expression level between HMGB3 and TGIF2 in the 21 pairs ESCC tissues and adjacent nontumor. E. Correlation analysis of the IHC score between HMGB3 and TGIF2 in the 57 pairs ESCC tissues and adjacent nontumor. F. Correlation analysis of HMGB3 and TGIF2 expression in ESCC tissues ( $\mathrm{N}=112) \mathrm{G}$. Kaplan-Meier analysis shows the relationship between HMGB3 mRNA expression and OS for the patients in the ESCC tissue microarray $(\mathrm{N}=112)$. H. Kaplan-Meier analysis shows the relationship between the combination of HMGB3 and TGIF2 mRNA expression and OS for the patients in the ESCC tissue microarray $(N=112)$. All the data are shown as the mean $\pm S D$. $* \star \star$ $P<0.001,{ }^{*} P<0.01$ and $* P<0.05$. 

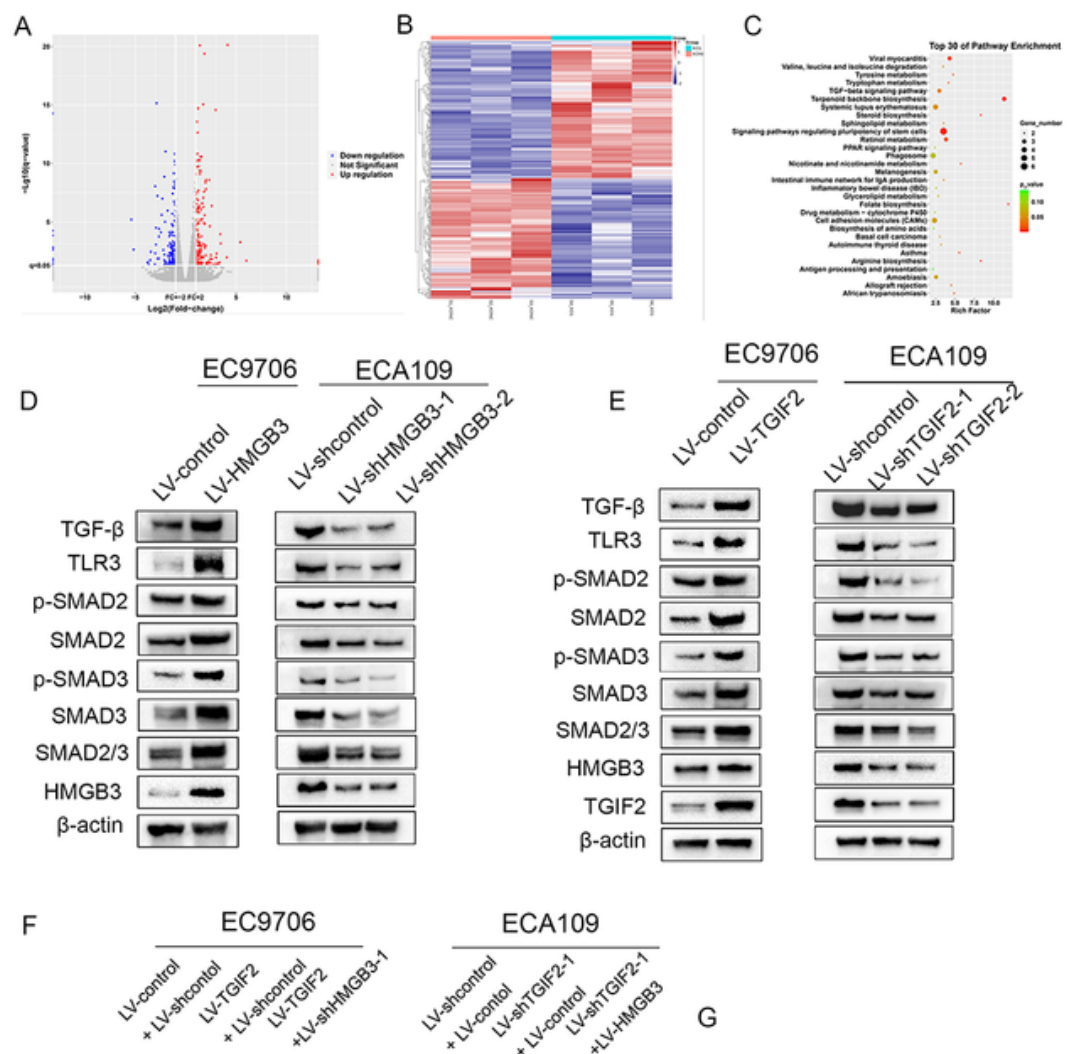

\begin{tabular}{|c|c|}
\hline TGF- $\beta$ & 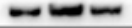 \\
\hline TLR3 & $-8=-$ \\
\hline p-SMAD2 & -- \\
\hline AAD2 & $-\infty-$ \\
\hline SMAD3 & $-\infty-$ \\
\hline SMAD3 & $-\cdots$ \\
\hline SMAD $2 / 3$ & $=0$ \\
\hline & $--\cdots$ \\
\hline & $\cdots-\cdots$ \\
\hline -actin & - \\
\hline
\end{tabular}
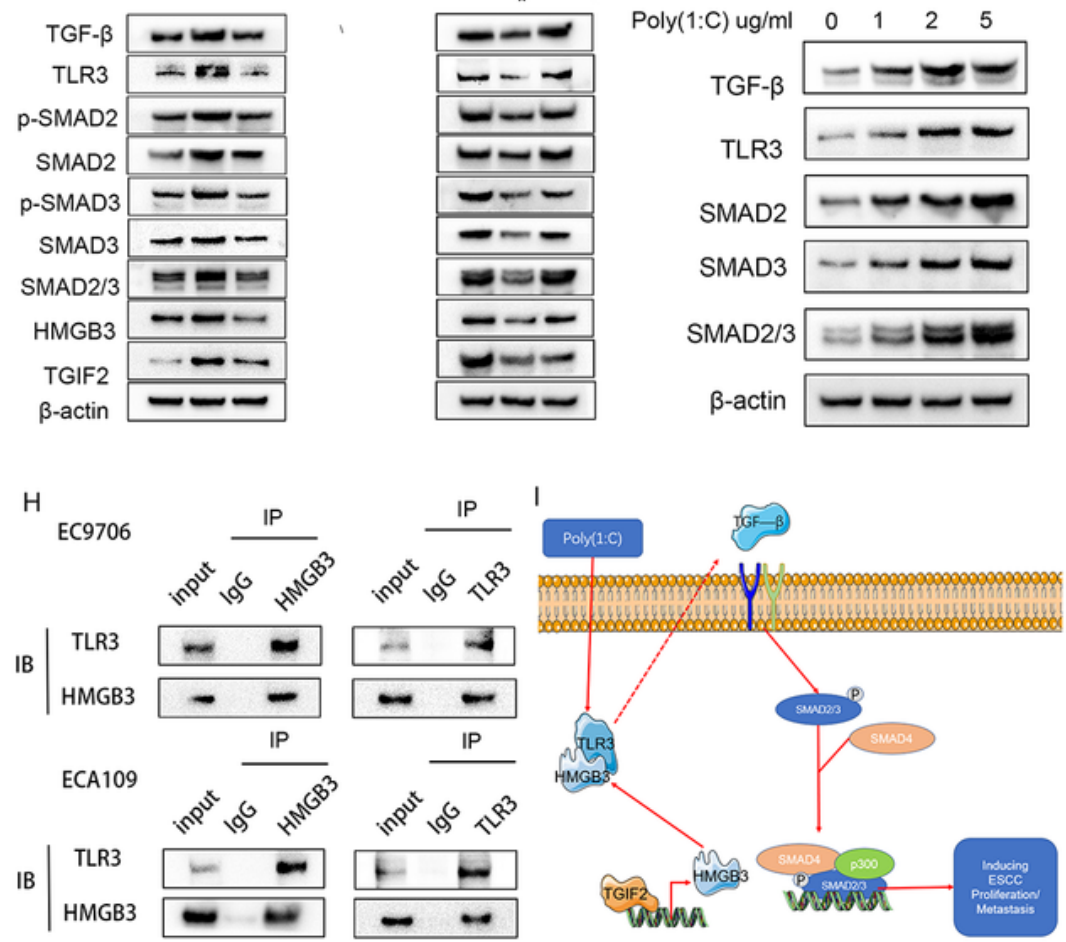

\section{Figure 8}

TGIF2 can positively regulate TGF- $\beta$ signaling in ESCC and this is HMGB3-dependent A. RNA-seq of LVcontrol vs LV-shHMGB3-1 in ECA109. Volcano plot of the RNA-seq shows the 168 upregulated genes and 141 downregulated ones. The blue dots indicate the lower expressed genes while the red dots indicate the higher expressed genes in ECA109-control compared with ECA109-shHMGB3-1. B. RNA-seq of LV-control vs LV-shHMGB3-1 in ECA109. Heatmap of the RNA-seq shows the 168 upregulated genes and 141 
downregulated ones. C. RNA-seq of LV-control vs LV-HMGB3-1 in ECA109. Heatmap of KEGG illustrates the correlation between different HMGB3 expression and relevant KEGG pathways. D. WB analysis shows the positive regulating effect of HMGB3 on Smad-dependent TGF- $\beta$. E. WB analysis shows the positive regulating effect of TGIF2 on Smad-dependent TGF- $\beta$. F. WB analysis shows the positive regulating effect of TGIF2 on Smad-dependent TGF- $\beta$ is HMGB3-dependent. G. Different concentration of poly (1:C) was performed in EC9706. WB analysis shows the positive regulating effect of TLR3 on Smad-dependent TGF- $\beta$. H. Co-IP test was performed to investigate the direct combination of HMGB3 and TLR3. I. A schematic model of the role of the TGIF2/HMGB3/TLR3 axis in inducing ESCC proliferation and metastasis.

\section{Supplementary Files}

This is a list of supplementary files associated with this preprint. Click to download.

- Additional1.jpg

- Additional2.jpg

- all.counts.KO1vsKONC.edgeRdifferential.xls 